\title{
DNA in a liquid-crystalline environment: Tight bends, rings, supercoils
}

\author{
Theo Odijk \\ Faculty of Chemical Engineering and Materials Science, Delft University of Technology, P.O. Box 5045, \\ 2600 GA Delft, The Netherlands
}

(Received 14 November 1995; accepted 10 April 1996)

\begin{abstract}
The entropy of tightly bent DNA is investigated in a variety of problems: closure probabilities, hairpin formation, nicked coils, plectonemic supercoiling, all in states with liquid-crystalline order. A new semiclassical method is presented for deriving the Green function of a tightly curved wormlike chain. Precise estimates for the entropy arising from undulations are given for tightly bent DNA in weak, intermediate, and strong nematic fields. A formal statistical mechanical analysis is outlined for hairpins and supercoils. The elongation of closed DNA without twist is computed in strong nematic fields. A scaling theory is given for a liquid crystal of untwisted DNA rings in which nematic order and ring elongation are self-consistently coupled. The elongation of plectonemic supercoils is evaluated for weak and strong nematic fields. The pitch of a cholesteric phase of plectonemic or loose supercoils is shown to be directly related to their writhe. (C) 1996 American
\end{abstract} Institute of Physics. [S0021-9606(96)50527-1]

\section{INTRODUCTION}

Tight curves of double-stranded DNA are found frequently in a wide variety of circumstances: in nucleosomes, in adsorbed states, when supercoiled, and in confined isotropic, cholesteric, and hexagonal phases. The orientational fluctuations of an unconstrained section of DNA viewed as a wormlike chain, are distributed as a Gaussian ${ }^{1}$ but the statistical mechanics of tightly bent DNA is a far less trivial problem. A classic example of the latter is supercoiled DNA which has routinely been studied by neglecting the entropy altogether. ${ }^{2-14}$ Often, this may be a very good approximation. Still, in certain experiments, the undulations of the DNA helix within supercoils do play a significant role. ${ }^{15-20}$ Theoretical approaches accounting for undulations include simulations, ${ }^{21-27}$ analytical work $^{28-34}$ and scaling analyses. ${ }^{35,36}$

The problem of entropy becomes more acute if the DNA is confined in an ordered state. The slight bending of a semiflexible chain in nematic ${ }^{37-40}$ and hexagonal phases ${ }^{41,42}$ is fairly well understood. The deflection length ${ }^{43}$ governed by nematic order, chain elasticity, and entropy, is independent of the deformation in this case. The opposite regime of tight bending is our interest here. Recently, the occurrence of tight curves like hairpins has been convincingly demonstrated in thermotropic polyesters. ${ }^{44}$ There is now a body of theoretical literature on hairpins, with and without the effects of entropy. ${ }^{45-54}$ Nevertheless, there is still a need for semiquantitative insight into the role of entropy in these and analogous types of problems.

The object of this paper is twofold: On the one hand, to compute the entropy of one tight bend of DNA in an orientationally ordered field in a fairly rigorous fashion (Sec. III); on the other, to attempt to understand on a qualitative level, complicated problems involving tightly curved sections of DNA under nematic stress which may be caused by the DNA itself (Secs. V to VIII). Section IV outlines several qualitative considerations employed in the latter class of problems.
Section V describes the elongation and rippling of a small DNA ring in a nematic field. In Sec. VI a suspension of such rings without twist is considered; the nematic field is induced by the excluded-volume effect between the rings. In Sec. VII, twist is taken into account by studying the extension and possible rippling of a plectonemic supercoil in a nematic. Recent experiments ${ }^{55}$ on plasmid DNA within bacterial cells allude to an intriguing relation between DNA topology and cholesteric order. This connection is understood by expressing the cholesteric pitch in terms of the writhe in a liquidcrystalline suspension packed with DNA supercoils.

\section{ENTROPY OF A TIGHT BEND IN THE ISOTROPIC STATE}

The configuration of a simple wormlike chain of contour length $l$ is described by the unit vector $\mathbf{u}(s)$ tangent to the curve, where $s$ is the contour distance from one end (Fig. 1). In our Cartesian coordinate system $(x, y, z)$, it is given in terms of the polar angles $\theta(s)$ and $\varphi(s)$

$\mathbf{u}(s)=[\sin \theta(s) \cos \varphi(s), \sin \theta(s) \sin \varphi(s), \cos \theta(s)]$.

The bending energy is a Hookean law ${ }^{56}$

$$
U_{b}=\frac{1}{2} P k_{B} T \int_{0}^{l} d s\left(\frac{d \mathbf{u}}{d s}\right)^{2},
$$

where a bending force constant is the product of the persistence length $P$ and the temperature $T\left(k_{B}=\right.$ Boltzmann's constant). The statistical mechanical quantity of interest is the partition function ${ }^{56} G$ with both ends of the chain fixed at $s=0$ and $s=l$ 


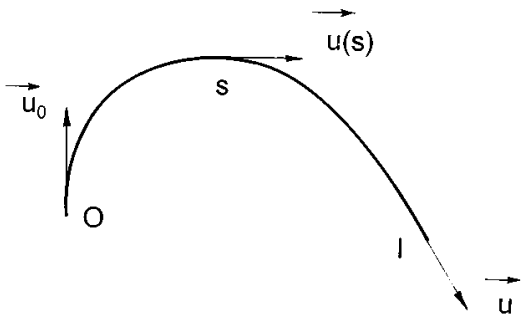

FIG. 1. One configuration of a tightly bent wormlike chain.

$$
\begin{aligned}
G\left(\mathbf{u}, l ; \mathbf{u}_{0}, 0\right)= & \int_{\mathbf{u}(0)=\mathbf{u}_{0}}^{\mathbf{u}(l)=\mathbf{u}} \mathscr{D}[\mathbf{u}(s)] \delta\left(\mathbf{u}^{2}(s)-1\right) \\
& \times \exp \left(-U_{b} / k_{B} T\right),
\end{aligned}
$$

which is the solution to ${ }^{56}$

$$
\frac{\partial G}{\partial l}-\frac{1}{2 P} \Delta_{\mathbf{u}} G=\delta(l) \delta\left(\mathbf{u}-\mathbf{u}_{0}\right)
$$

with $\Delta_{\mathbf{u}}$ the Laplacian on the unit sphere, so that $G$ is a Green function.

We would like to evaluate $G$ for tight bends $(L \equiv l / 2 P$ $<1)$. One option is to compute Eq. (2.3) in the semiclassical limit but how does one deal with the nontrivial functional integral (2.3)? (See below.) Another is to manipulate the solution to Eq. (2.4) which is a bilinear expansion in terms of the Legendre polynomials $P_{n}(\cos \theta)$. For instance, for a worm starting in the $z$ direction we have $\mathrm{e}^{56}$

$$
\begin{aligned}
G(\theta, \varphi, l ; 0,0,0) \\
\quad=\frac{1}{4 \pi} \sum_{n=0}^{\infty}(2 n+1) P_{n}(1) P_{n}(\cos \theta) e^{-n(n+1) L} .
\end{aligned}
$$

In the classical limit, $P \rightarrow \infty$ or $L \rightarrow 0$ formally; hence, in the semiclassical limit (Fig. 1), we would like to sum the terms in Eq. (2.5) since the exponential decays only when $n^{2} L=\mathcal{Q}(1)$ at least. But a naive summation is analytically useless for $P_{n}(\cos \theta)$ is a rapidly oscillating function of $n$.

Experience with this type of summation has arisen from semiclassical computations in quantum mechanics. A quantum particle scattered in a tight orbit is analogous to a tightly bent chain undergoing thermal undulations; the limit Planck's constant $\hbar \rightarrow 0$ in the former is similar to the limit $P \rightarrow \infty$ in the latter. Berry and Mount have elegantly summarized the complex history of semiclassical mechanics as developed by many theoreticians of repute.$^{57}$ In particular, the usual WKB approximation is often deficient because it is not always uniformly valid. It is rendered uniformly so with the help of the Langer modification, ${ }^{58}$ in which the angular quantum number $-l$ in the quantum literature-is replaced by $l-$ $\frac{1}{2}$ (in our case this number is denoted by $n$ ). Moreover, the modified WKB approximation is also more accurate to higher order in most problems. This is especially important in our computations of the entropy of tight bends [the bending energy is of $\mathscr{O}\left(L^{-1}\right)$ but it turns out that the entropy is of $Q(L)$, i.e., a second order term, in the argument of exponential terms contained in the probability functions]. In the end,

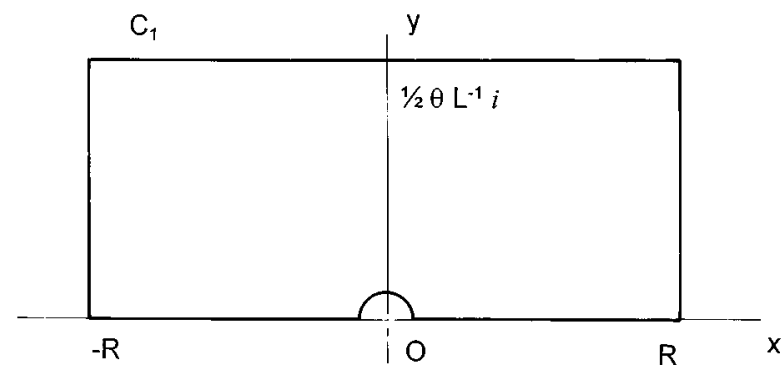

FIG. 2. The contour $C_{1}(z=x+i y)$.

Berry and Mount ${ }^{57}$ advocate the use of the following Poisson summation formula since it automatically accounts for the Langer modification:

$$
\sum_{n=0}^{\infty} u(n)=\sum_{M=-\infty}^{\infty} e^{-i M \pi} \int_{0}^{\infty} d n u\left(n-\frac{1}{2}\right) e^{2 \pi i M n} .
$$

Here, the winding number $M$ is the number of times the classical path encircles the origin. In our case, we set $\hbar \equiv 1$ formally. In order to evaluate the semiclassical limit, one approximates the eigenfunction within the form $u(n)$ by its asymptotic representation at large $n$ (i.e., in the modified WKB approximation). Wild oscillations are circumvented by using the theory of functions of a complex variable. For instance, in an intermediate regime for the problem at hand we have $\mathrm{e}^{57}$

$$
\begin{aligned}
& P_{n-(1 / 2)}(\cos \theta) \simeq\left(\frac{2}{\pi n \sin \theta}\right)^{1 / 2} \cos \left(n \theta-\frac{\pi}{4}\right) \\
& \left(n^{-1} \lesssim \theta \lesssim \pi-n^{-1}\right) .
\end{aligned}
$$

Here focus is on the case $M=0$, so that

$$
\begin{aligned}
G \simeq & \left(\frac{1}{4 \pi^{3} \sin \theta}\right)^{1 / 2} e^{(1 / 4) L} \int_{0}^{\infty} d n n^{1 / 2} e^{-n^{2} L} \\
& \times(\cos n \theta+\sin \dot{n} \theta) .
\end{aligned}
$$

In order to integrate Eq. (2.8), consider the contour intergal $I_{1}$, in the complex plane

$$
\begin{aligned}
& I_{1}=\oint_{C_{1}} d z z^{1 / 2} e^{f_{1}(z)}, \\
& \begin{aligned}
f_{1}(z) & =-L z^{2}+\theta z i \\
& =-\frac{\theta^{2}}{4 L}-L\left(z-\frac{\theta i}{2 L}\right)^{2} .
\end{aligned}
\end{aligned}
$$

The integrand is analytic within the area enclosed by the rectangular contour $C_{1}$ (see Fig. 2). An asymptotic analysis of $I_{1}$ on the upper contour is possible for $\theta^{2} \gtrsim L$ as $R_{1} \rightarrow \infty$ and $L \rightarrow 0$. With the help of Cauchy's theorem, the leading term for the partition function becomes

$$
G(\theta) \sim \frac{1}{4 \pi}\left(\frac{\theta}{\sin \theta}\right)^{1 / 2} L^{-1} \exp \left(-\frac{\theta^{2}}{4 L}+\frac{1}{4} L\right) .
$$


As $L \rightarrow 0$ and $\theta^{2} \simeq L$, this joins smoothly to the usual Gaussian distribution. ${ }^{59}$ Moreover, as we approach its outer edge of validity $\left(\theta \rightarrow \pi-L^{1 / 2} ;|z|=\mathcal{Q}\left(L^{-1}\right)\right.$, recall that the dominant region in Eq. (2.8) is $0 \leqslant n \leqslant L^{-1 / 2}$ ), we regain, to order unity, an analytical result due to Shimada and Yamakawa ${ }^{29}$

$$
G(\pi)=\frac{1}{4} \pi^{1 / 2} L^{-3 / 2} \exp \left(-\frac{\pi^{2}}{4 L}+\frac{1}{4} L\right) .
$$

They also computed various ring-closure probabilities similar in form to this expression by a discrete summation of functional integrals like Eq. (2.3) around the classical paths. Considerable effort has been devoted to developing semiclassical approximations to Feynman path integrals (see, for instance, Refs. 57, 60-62). Gutzwiller has tried to build such approximations from ideas imbedded in classical mechanics. Here, the calculation of the classical path is circumvented altogether which may be advantageous for wormlike chains.

Next, we analyze the properties of the tightly bent worm described by Eq. (2.11). The free energy of the orientationally constrained bend may be written as

$$
\begin{aligned}
F(\theta) / k_{B} T & =-\ln G(\theta) \\
& =\frac{\theta^{2} P}{2 l}+\ln \frac{2 \pi l}{P}-\frac{l}{8 P}+\frac{1}{2} \ln \frac{\sin \theta}{\theta} .
\end{aligned}
$$

Equation (2.3) yields the average bending energy

$$
\begin{aligned}
\frac{\left\langle U_{b}(\theta)\right\rangle}{k_{B} T} & =-P \frac{\partial \ln G(\theta)}{\partial P} \\
& =\frac{\theta^{2} P}{2 l}-1+\frac{l}{8 P}
\end{aligned}
$$

so that the entropy is given by

$$
k_{B}^{-1} S(\theta)=\ln \left(\frac{P}{l e}\right)+\frac{l}{4 P}+\frac{1}{2} \ln \frac{\theta}{\sin \theta} .
$$

Hence, the entropy is only a weak function of the angular restriction $\theta$ and very close to the value it would have if it were in the Gaussian limit, which is strictly valid for $\theta \ll 1$. Physically, Eq. (2.15) implies undulations are virtually unaffected by the degree of curvature, at least in this orientationally constrained problem. Tight bending does not freeze out undulations in contradiction with my earlier claim. ${ }^{35}$ It is also illuminating to inspect the average curvature (or its moments)

$$
\begin{aligned}
\left\langle R^{-2}\right\rangle & =\frac{2\left\langle U_{b}(\theta)\right\rangle}{l P k_{B} T} \\
& =R_{c}^{-2}-\frac{2}{l P}+\frac{1}{4 P^{2}},
\end{aligned}
$$

where $R$ is the radius of curvature of the bend and $R_{c}$ is its value in the classical limit $(P \rightarrow \infty)$. Upon introducing an angular fluctuation

$$
\left\langle R^{-2}\right\rangle^{1 / 2} \equiv R_{c}\left(1+\left\langle\Delta \theta^{2}\right\rangle\right)
$$

we get

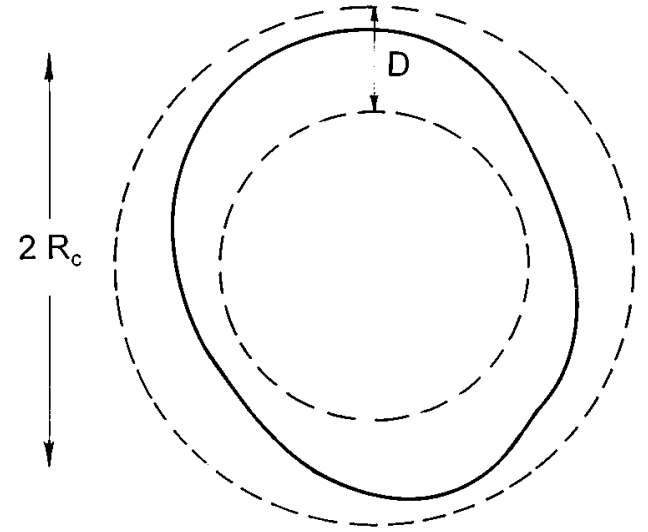

FIG. 3. A tight wormlike ring has a fuzzy diameter $D$.

$$
\left\langle\Delta \theta^{2}\right\rangle \simeq \frac{R_{c}^{2}}{l P}=\frac{l}{\theta^{2} P} .
$$

The curvature and the radius of curvature are weighted differently so one expects a circular wormlike ring to shrink when the undulations are switched on: $\langle R\rangle \simeq R_{c}\left(1-\left\langle\Delta \theta^{2}\right\rangle\right)$. The ring has a fuzzy diameter $D \simeq R_{c}\left\langle\Delta \theta^{2}\right\rangle^{1 / 2}$ (see Fig. 3)

$$
\begin{aligned}
& \left\langle\Delta \theta^{2}\right\rangle \simeq \frac{R_{c}}{P} \\
& D \simeq R_{c}^{3 / 2} P^{-1 / 2}
\end{aligned}
$$

which disagrees with Ref. 35 for obvious reasons, but agrees with analytical work by Shimada and Yamakawa ${ }^{25}$ and Monte Carlo simulations by Camacho, Fisher, and Singh. ${ }^{26}$ The latter authors assumed that tight bending might not interfere too much with chain undulations and they went on to derive Eq. (2.20) using fluctuation theory.

Another heuristic way of understanding Eq. (2.19) is by supposing the undulations are caused by a tension $f_{c}$ within the ring. This ought to be related to the classical bending energy $U_{b}=k_{B} T l P / 2 R_{c}^{2}=2 \pi^{2} P k_{B} T / l$ by

$$
f_{c} \simeq-\frac{\partial U_{b}}{\partial l}=2 \pi^{2} k_{B} T P l^{-2}
$$

But there are fluctuations expressed by the angle $\Delta \boldsymbol{\theta}=\left(\Delta \theta_{1}(s), \Delta \theta_{2}(s)\right)$, where the indices denote directions orthogonal to the local tangent $\mathbf{u}(s)$. Neglecting curvature as argued above, we may write for the Hamiltonian

$$
\begin{aligned}
\mathscr{H}= & \frac{1}{2} P k_{B} T \int_{0}^{l} d s\left[\left(\frac{d \Delta \theta_{1}}{d s}\right)^{2}+\left(\frac{d \Delta \theta_{2}}{d s}\right)^{2}\right] \\
& +\frac{1}{2} f_{c} \int_{0}^{l} d s\left(\Delta \theta_{1}^{2}+\Delta \theta_{2}^{2}\right),
\end{aligned}
$$

utilizing an expression for the effective length 


$$
\begin{aligned}
l_{\mathrm{eff}} & \simeq \int_{0}^{l} d s\langle\cos |\Delta \boldsymbol{\theta}|\rangle \\
& \simeq l\left(1-\frac{1}{2}\left\langle\Delta \theta_{1}^{2}\right\rangle-\frac{1}{2}\left\langle\Delta \theta_{2}^{2}\right\rangle\right) .
\end{aligned}
$$

Equation (2.22) is a quantum harmonic oscillator which is easily solved for long chains ${ }^{63}$ by assuming the fluctuations are Gaussian

$$
g \sim \frac{\alpha_{0}}{2 \pi} \exp -\frac{1}{2} \alpha_{0} \Delta \theta^{2} \quad\left(\alpha_{0} \gg 1\right) .
$$

The total free energy becomes ${ }^{63}$

$$
\Delta F_{\mathrm{tot}}=\frac{l \alpha_{0} k_{B} T}{4 P}+\frac{f_{c} l}{\alpha_{0}}
$$

which upon minimization gives

$$
\begin{aligned}
& \alpha_{0}=2\left(P f_{c} / k_{B} T\right)^{1 / 2} \\
& \left\langle\Delta \theta^{2}\right\rangle=\frac{2}{\alpha_{0}}=\frac{l}{2^{1 / 2} \pi P}
\end{aligned}
$$

The latter equation shows that end effects may be safely neglected. ${ }^{63}$ The deflection length ${ }^{63}$ is

$$
\lambda=P / \alpha_{0} \simeq l .
$$

Equation (2.28) means the ring is deforming globally under the influence of thermal excitations. Hence, there are large fluctuations in the curvature. The assumption of a more or less constant curvature implicit in Ref. 35 is erroneous.

\section{TIGHT BEND IN A NEMATIC}

Next we investigate how the entropy of a tight constrained bend is affected when it is put in a nematic liquid crystal (Fig. 4). If we assume the nematic itself is unperturbed by the chain, which is not entirely true, ${ }^{46,54}$ we may write for the nematic potential exerted on the chain

$$
\begin{aligned}
& U_{n}[\mathbf{u}(s)]=\frac{k_{B} T}{2 P} \int_{0}^{l} d s V(\mathbf{n} \cdot \mathbf{u}(s)), \\
& V(\cos \theta)=V(-\cos \theta),
\end{aligned}
$$

where the director has been chosen to point in the $z$ direction. The function $V$ is dimensionless and depends on the nematic order of the solvent. A very convenient choice depending on one parameter only is ${ }^{46}$

$$
V=\Gamma \sin ^{2} \theta
$$

At low $\Gamma$, this is basically $P_{2}(\cos \theta)$ whereas at high $\Gamma, V$ behaves essentially like a harmonic form $\Gamma \theta^{2}$ owing to Boltzmann weighting. In the latter case, the orientational distribution will simplify to a Gaussian; ${ }^{43}$ the order parameter $S_{n}$ is simply connected to the coupling parameter $\Gamma$

$$
\begin{aligned}
& g \sim \frac{\alpha}{4 \pi} \exp -\frac{1}{2} \alpha \theta^{2} \quad\left(\alpha \gg 1 ; 0 \leqslant \theta<\frac{1}{2} \pi\right), \\
& g(\theta)=g(\pi-\theta) \quad\left\langle\theta^{2}\right\rangle \sim 2 \alpha^{-1}, \\
& S_{n} \equiv\left\langle P_{2}(\cos \theta)\right\rangle \sim 1-3 \alpha^{-1},
\end{aligned}
$$

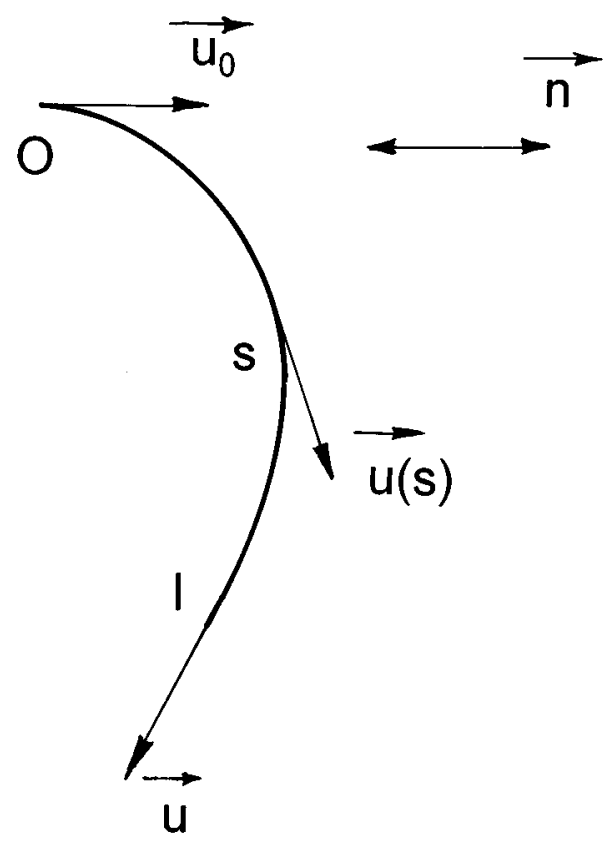

FIG. 4. A tight bend in a nematic.

$$
\Gamma=\frac{1}{4} \alpha^{2} .
$$

Adding Eq. (3.1) to the bending energy [Eq. (2.2)] and expanding the new partition function [Eq. (2.3)], we get

$$
\begin{aligned}
& \frac{\partial G}{\partial l}-\frac{1}{2 P} \mathscr{D}_{\theta} G+\frac{1}{2 P} V G=\delta(l) \delta(\cos \theta), \\
& \mathscr{D}_{\theta} \equiv \frac{1}{\sin \theta} \frac{\partial}{\partial \theta}\left(\sin \theta \frac{\partial}{\partial \theta}\right) .
\end{aligned}
$$

The Green function $G$ may be expanded in terms of orthonormal eigenfunctions $\varphi_{n}$

$$
G(\theta, l ; 0,0)=\sum_{n} \varphi_{n}^{*}(1) \varphi_{n}(\cos \theta) e^{-E_{n} L}
$$

where the eigenvalues have to be determined from

$$
-\mathscr{L}_{\theta} \varphi_{n}+V(\cos \theta) \varphi_{n}=E_{n} \varphi_{n}
$$

\section{A. Semiclassical limit for weak and intermediate fields}

According to the prescription of Sec. II, we first need an appropriate asymptotic formula for the eigenfunction $\varphi_{n}$, but also a sufficiently accurate estimate for $E_{n}$. These can be computed in the WKB approximation provided one uses a Langer modification. ${ }^{58}$ The reasoning used here is similar in spirit to that adopted by Landau and Lifshitz in their semiclassical evaluation of the Legendre polynomials. ${ }^{64}$ One first introduces $\chi_{n}=\varphi_{n} \sin ^{1 / 2} \theta$ into Eq. (3.10)

$$
\frac{d^{2} \chi_{n}}{d \theta^{2}}+\left(E_{n}+\frac{1}{4}+\frac{1}{4 \sin ^{2} \theta}-V\right) \chi_{n}=0 .
$$


In the semiclassical limit, $L$ is very small so at fixed constraints we first focus on the regime with $\theta^{2} E_{n} \gg 1$ and $(\pi-\theta)^{2} E_{n} \gg 1$. Herein, Eq. (3.11) is approximated by

$$
\frac{d^{2} \chi_{n}}{d \theta^{2}}+\left(\bar{E}_{n}-V\right) \chi_{n}=0,
$$

where we introduce a Langer modification ${ }^{57,58} \bar{E}_{n}=E_{n}+a_{1}$ with $a_{1}$ a constant supposedly independent of $V$. Upon introducing the "momentum"

$$
p \equiv\left(\bar{E}_{n}-V\right)^{1 / 2}
$$

with $\bar{E}_{n}>V$ for all angles, we may now write a WKB approximation $^{64}$ for $\chi_{n}$ or $\varphi_{n}$

$$
\varphi_{n}=\left(\frac{C_{1}}{p^{1 / 2} \sin ^{1 / 2} \theta}\right) \sin \left[\int_{0}^{\theta} d \theta p(\theta)+C_{2}\right],
$$

where $C_{1}$ and $C_{2}$ are constants independent of $V$. Actually, this expression turns out to be uniformly valid right down to small $\theta$. For small $\theta$, the eigenvalue equation (3.10) may be expressed as

$$
\frac{d^{2} \varphi_{n}}{d \theta^{2}}+\frac{1}{\theta} \frac{d \varphi_{n}}{d \theta}+\left(E_{n}+a_{2}\right) \varphi_{n}=0
$$

where, again, we introduce a Langer modification. ${ }^{64}$ The solution to this is a Bessel function $J_{0}$ of order zero whose behavior at large $E_{n}^{1 / 2} \theta$ is given by

$$
\varphi_{n} \sim C_{3}\left(\frac{2}{\pi n \theta}\right)^{1 / 2} \sin \left[\left(E_{n}+a_{2}\right)^{1 / 2} \theta+\frac{1}{4} \pi\right]
$$

with $C_{3}$ a constant.

Equations (3.14) and (3.16) overlap provided $a_{1}=a_{2}$ and $c_{2}=\frac{1}{4} \pi$.

Next, symmetry arguments establish the eigenvalues: Those pertinent to the symmetric eigenfunctions $\left(\varphi_{n}(\theta)=\varphi_{n}(\pi-\theta)\right)$ obey

$$
\int_{0}^{\pi} d \theta p^{1 / 2}=\left(n+\frac{1}{2}\right) \pi
$$

with $n$ even, while an identical relation with $n$ odd holds for antisymmetric eigenstates $\left(\varphi_{n}(\theta)=-\varphi_{n}(\pi-\theta)\right)$. Finally, for ideal chains $(V=0)$, one has

$$
\begin{aligned}
& \mathscr{L}_{\theta} P_{n}(\cos \theta)=-E_{n}^{(0)} P_{n}(\cos \theta), \\
& E_{n}^{(0)}=n(n+1)
\end{aligned}
$$

which fixes the constant $a_{1}=\frac{1}{4}$.

Let us now compute the entropy of a tight wormlike bend in a nematic potential given by Eq. (3.2) which is sufficiently general for our purposes. Then, the eigenvalue relation [Eq. (3.17)] is given in terms of a complete elliptic integral $^{65}$ of the second kind with modulus $q$

$$
\begin{aligned}
& \mathscr{E}(w) \equiv \int_{0}^{\pi / 2} d \theta\left(1-w^{2} \sin ^{2} \theta\right)^{1 / 2}, \\
& \left(E_{n}+\frac{1}{4}\right)^{1 / 2} \mathscr{E}(q)=\left(\frac{1}{2} n+\frac{1}{4}\right) \pi, \\
& q^{2}=\Gamma /\left(E_{n}+\frac{1}{4}\right) .
\end{aligned}
$$

The accuracy of Eq. (3.21) can be gauged by comparing it, at small $q$, with standard perturbation theory ${ }^{64}$ to order $\Gamma$ (Hamiltonian $\mathscr{H}=\mathscr{H}_{0}+V ; \mathscr{H}_{0}=-\mathscr{C}_{\theta}$ )

$$
\begin{aligned}
& E_{n}=E_{n}^{(0)}+\frac{1}{2} \Gamma, \\
& n=0,1,2, \ldots \quad(\text { current } \mathrm{WKB}), \\
& E_{n}=E_{n}^{(0)}+\frac{1}{2} \Gamma-\frac{\Gamma}{2\left(4 n^{2}+4 n-3\right)} \\
& n=0,1,2, \ldots \quad \text { (perturbation theory), }
\end{aligned}
$$

where the following relation has been employed: ${ }^{48}$

$$
\int_{-1}^{1} d x P_{2}(x) P_{n}^{2}(x)=\frac{2 n(n+1)}{(2 n+3)(2 n+1)(2 n-3)} \text {. }
$$

The present WKB theory is very good even for $n$ as low as 2 . There should be no problem in the semiclassical limit $(n \gg 1)$.

The free energy of a semicircular bend is now evaluated $(\theta=\pi)$. An analysis of Eqs. (3.9) and (3.14) shows that we need to consider

$$
G(\pi, l ; 0,0) \sim \sum_{n=0}^{\infty} n(-1)^{n} e^{-E_{n} L} .
$$

At this stage, it is expedient to introduce the function $\mathscr{E}^{A}(q)$, which has the following properties:

(i) $\mathscr{E}^{A}(q) \equiv \mathscr{E}(q)$ for $q \leqslant c_{4} q_{*}$ with $2 c_{4} q_{*}<1$; $q_{*}^{2}=\mathscr{O}\left(\Gamma L^{2}\right)$ and $c_{4}=\odot(5)$ say.

(ii) $\mathscr{E}^{A}(q)$ tends to the limit $\mathscr{E}\left(2 c_{4} q_{*}\right)$ for $c_{4} q_{*}<q$ $<\infty ; \mathscr{E}^{A}(q)$ is continuous and continuously differentiable.

(iii) $\mathscr{E}^{A}(z)$ is analytic in the entire complex plane.

Note that $\mathscr{E}^{A}$ may be useful even for intermediate nematic fields, for $\Gamma$ may be greater than unity as $L$ becomes very small. Since $E_{n}$ is typically $\mathscr{O}\left(L^{-1}\right)$, Eq. (3.26) may be approximated by

$$
G \simeq-i \int_{0}^{\infty} d n n e^{n \pi i-L E_{n-(1 / 2)}^{A}}
$$

where a Langer modification has been accounted for and $E^{A}$ is given by Eq. (3.21), but with $\mathscr{E}^{A}$ instead of $\mathscr{E}$. Therefore, we next focus on the contour integral $I_{2}$ in the complex plane where $C_{1}$ is the same contour introduced in Sec. II, although the origin need not be excluded (see Fig. 2).

$$
\begin{aligned}
& I_{2}=\oint_{c_{1}} d z z e^{f_{2}(z)}, \\
& f_{2}(z)=\pi z i-E^{A}(z) L, \\
& \left(E^{A}(z)+\frac{1}{4}\right)^{1 / 2} \mathscr{E}^{A}(q)=\frac{1}{2} \pi z, \\
& q^{2}=\Gamma /\left(E^{A}(z)+\frac{1}{4}\right) .
\end{aligned}
$$

Note that $\mathscr{E}^{A}$ is a very slowly varying function of $z$ so that terms of higher order than the second are minute in the semiclassical limit. 


$$
\begin{aligned}
f_{2}(z)= & \frac{1}{4} L-2 \mathscr{K}\left(q_{0}\right) \mathscr{E}\left(q_{0}\right) L^{-1}+\mathscr{K}^{2}\left(q_{0}\right) L^{-1} \\
& -\frac{1}{2} E^{\prime \prime}\left(z_{0}\right) L\left(z-z_{0}\right)^{2}+\cdots .
\end{aligned}
$$

Here, $z_{0}$ is purely imaginary

$$
z_{0}=\frac{2 \mathscr{K}\left(q_{0}\right) \mathscr{E}\left(q_{0}\right) i}{\pi L}
$$

$\mathscr{K}$ is the complete elliptic integral of the first kind ${ }^{65}$

$$
\mathscr{K}(w)=\int_{0}^{\pi / 2} d \theta\left(1-w^{2} \sin ^{2} \theta\right)^{-1 / 2} .
$$

$q_{0}$ is a function only of the group $\Gamma L^{2}$

$$
q_{0}^{2}=-\frac{L^{2} \Gamma}{\mathscr{K}^{2}\left(q_{0}\right)}
$$

and both elliptic integrals have been continued analytically. Hence, the free energy of the tight bend becomes

$$
F / k_{B} T \simeq 4 \mathscr{K}\left(q_{0}\right) \mathscr{E}\left(q_{0}\right) P l^{-1}-2 \mathscr{K}^{2}\left(q_{0}\right) P l^{-1}-l / 8 P .
$$

From the definition of the partition function, we finally get the entropy

$$
S=\left.\frac{P}{T} \frac{\partial F}{\partial P}\right|_{\Gamma P^{-2} \text { fixed }}-\frac{F}{T}=\frac{l k_{B}}{4 P} .
$$

The entropy has thus been computed without solving the Euler-Lagrange equation for the path of minimum energy. To conclude, the entropy is not influenced by the nematic field [or very weakly since several very small higher order terms have been deleted; the first term of Eq. (2.15) is absent because nonexponential prefactors have been disregarded above].

\section{B. Strong fields}

The analysis of the previous section breaks down as the coupling constant $\Gamma$ reaches $L^{-2}$. The regime $\Gamma L^{2}=Q(1)$ is complicated but strong fields $\left(\Gamma L^{2} \gg 1\right)$ can be handled by WKB methods again. There now exists a range of angles for which $\bar{E}_{n}<V$. In the quantum analogy this is called tunneling ${ }^{64}$ because a particle does not have enough energy to surmount the barrier in the sense of classical mechanics, although quantum-mechanically the probability of doing so is nonzero. Warner et al. have already discussed the nematic hairpin as a barrier problem in the WKB approximation at some length. ${ }^{47}$ The problem is reanalyzed for a finite bend so as to stress several features pertinent here.

If the bend is less than a persistence length long $(l<P)$ the nematic coupling parameter $\Gamma$ will be much greater than unity. Equation (3.12) is now valid for all $n$ including the ground state $n=0$, provided $\theta>\theta_{0}\left[\theta_{0}=\mathscr{Q}\left(\Gamma^{1 / 4}\right)\right]$. The turning points $\theta_{1}$ and $\theta_{2}=\pi-\theta_{1}$ are defined by setting the momentum [Eq. (3.13)] equal to zero. We next solve Eq. (3.12) in a WKB approximation. A possible solution can be written as three smoothly matching approximations in three respective regimes $^{64}$

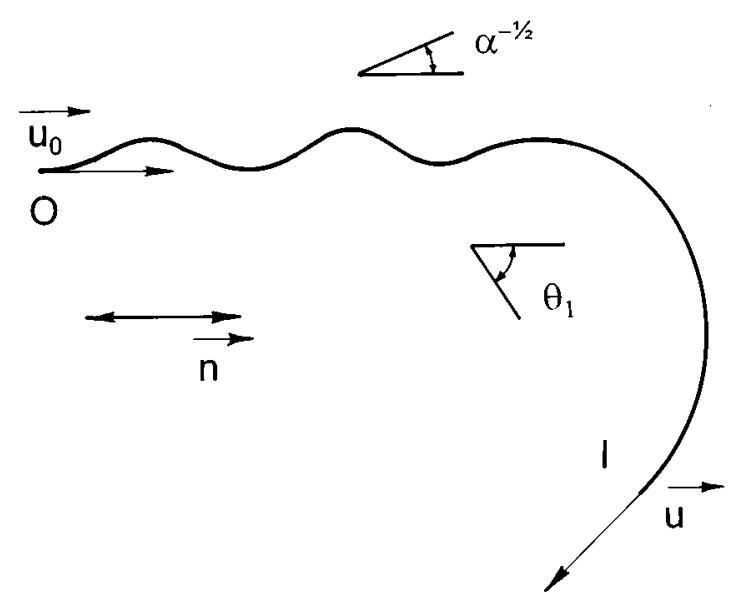

FIG. 5. A tight bend is partly rippled in a strong nematic field.

$$
\begin{aligned}
& \Psi_{n}=\left(\frac{2^{1 / 2} H}{p^{1 / 2}}\right) \sin \left[\int_{0}^{\theta} d \theta p+\frac{1}{4} \pi\right] \\
& \left(\theta_{0}<\theta<\theta_{1} ; \bar{E}_{n}>V\right), \\
& \Psi_{n}=\left(\frac{H}{2^{1 / 2} p^{1 / 2}}\right) \exp -\int_{\theta_{1}}^{\theta} d \theta|p| \\
& \left(\theta_{1}<\theta<\pi-\theta_{1} ; \bar{E}_{n}<V\right), \\
& \Psi_{n}=\left(\frac{H}{2^{1 / 2} p^{1 / 2}}\right) \exp \left[-\int_{\theta_{1}}^{\theta_{2}} d \theta|p|+\frac{1}{4} \pi i+\int_{\theta_{2}}^{\theta} d \theta p\right] \\
& \left(\theta_{2}<\theta<\pi-\theta_{0} ; \bar{E}_{n}>V\right),
\end{aligned}
$$

where $H$ is a normalization constant. These expressions do not represent a solution as such, for $\Psi_{n}$ does not have the requisite symmetry. Because the potential barrier is equivalent to two symmetric wells, the eigenfunctions must be symmetric and antisymmetric ${ }^{64}$

$$
\begin{gathered}
\chi_{n}^{s}(\theta)=2^{-1 / 2}\left(\Psi_{n}(\theta)+\Psi_{n}(\pi-\theta)\right) \\
n=0,2,4, \ldots . \\
\chi_{n}^{A s}(\theta)=2^{-1 / 2}\left(\Psi_{n}(\theta)-\Psi_{n}(\pi-\theta)\right) \\
n=1,3,5, \ldots .
\end{gathered}
$$

The splitting ${ }^{64}$ of the respective eigenvalues is exponentially small for $\Gamma \gg 1$ causing the spectrum to be almost degenerate

$$
\begin{aligned}
E_{n}^{A S}-E_{n}^{S} & =4 \Psi_{n}\left(\frac{1}{2} \pi\right) \Psi_{n}^{\prime}\left(\frac{1}{2} \pi\right) \\
& =\text { const. } \cdot \exp -\int_{\theta_{1}}^{\theta_{2}} d \theta|p| .
\end{aligned}
$$

The Green function [Eq. (3.9)] in the WKB approximation is real.

Let us focus on a tight bend with one end parallel to the nematic director which is pointing in the $z$ direction, and the other end fixed at orientation $\theta$ with $\theta_{1}<\theta<\theta_{2}$ (Fig. 5). For $\Gamma \gg 1$, the eigenvalues can be established in the WKB ap- 


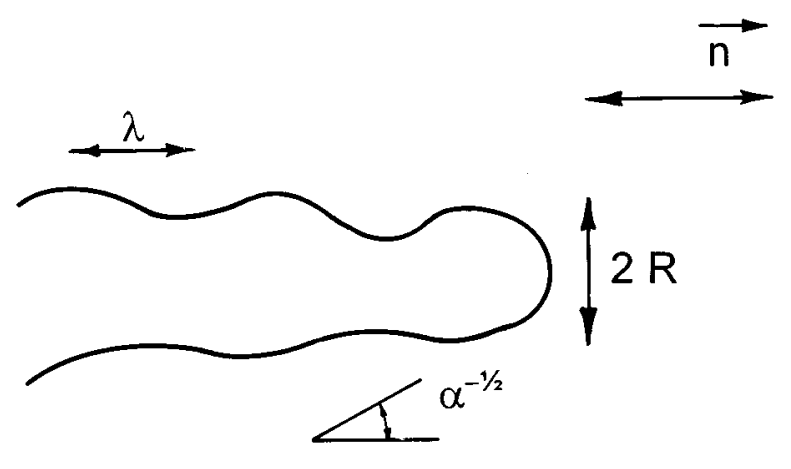

FIG. 6. A hairpin of radius $R$.

proximation by the Bohr-Sommerfeld condition, ${ }^{64}$ pertaining to a one dimensional well in the classically allowed regime $0<\theta<\theta_{1}$,

$$
\int_{0}^{\theta_{1}} d \theta p=\left(m+\frac{1}{2}\right) \pi
$$

The case $m=0$ corresponds to the states $n=0$ and $n=1$, the case $m=1$ to $n=2$ and $n=3$, and so forth. In the limit of strong fields, we have $\theta_{1} \simeq\left(\bar{E}_{n} / \Gamma\right)^{1 / 2}$ and Eq. (3.44) reduces to

$$
E_{n} \simeq(4 m+2) \Gamma^{1 / 2} .
$$

Accordingly, in the Green function only the two lowest states dominate in view of the condition $\Gamma L^{2} \gg 1$. Hence, the free energy associated with the two Boltzmann factors is

$$
F / k_{B} T \simeq 2 \Gamma^{1 / 2} L+\int_{\theta_{1}}^{\theta} d \theta\left(\Gamma \sin ^{2} \theta-\bar{E}_{0}\right)^{1 / 2} .
$$

In the limit of strong nematic fields, Eq. (3.6) holds. Therefore, the first term in Eq. (3.46) is precisely the free energy of a chain of length $l$ in a Gaussian nematic field, the chain being rippled on the scale of a deflection length. ${ }^{43}$ The contour length of this section is almost $l$ since the unrippled bend $\left(\theta>\theta_{1}\right)$ is very short (Fig. 5). This is a result of the requirement $\Gamma L^{2} \gg 1$ employed in the WKB approximation. The energy of the unrippled curve is given by the second term in Eq. (3.46), which is that of the classical path from $\theta_{1}$ to $\theta$. It scales as $\Gamma^{1 / 2}$ implying that the radius of curvature of this bend is $P \Gamma^{-1 / 2}$. The crossover between the two regimes is at $\theta_{1} \simeq \alpha^{-1 / 2}$.

\section{Self-consistent field theory of hairpins}

The entropy can be incorporated exactly in a selfconsistent field theory of hairpin formation for infinitely long chains (Fig. 6). We must be prepared to neglect long-range perturbations in the director fields $s^{46,54}$ in this type of approximation. Khokhlov and Semenov ${ }^{45}$ first proposed a selfconsistent field theory for hairpins arising in a nematic of hard cylindrical wormlike chains. Their theory was reanalyzed by Vroege and Odijk ${ }^{48}$ who gave an exact expression for the global persistence length-a measure of the typical distance between hairpins-in terms of the orientational distribution. In both works, the second virial approximation is adopted which is probably often a rather severe constraint when applied to concentrated solutions of DNA. ${ }^{66-68}$ Here, a theory for general self-consistent fields is presented which is much less restrictive.

A test chain of extremely long contour length $l$ has an orientational distribution $f(\mathbf{u})$ defined with respect to the director, the average direction of all the wormlike chains. The director is assumed to be uniform and fixed in space in the $z$ direction; in a nematic state $f(\mathbf{u})=f(\mathbf{u} \cdot \mathbf{n})=f(\tau)=f(-\tau)$ with $\mathbf{u}$ written in the form of Eq. (2.1) and $\tau \equiv \cos \theta$. The free energy of orientational confinement of the test chain is ${ }^{45}$

$$
F_{\text {or }}=-\frac{l k_{B} T}{2 P} \int d \mathbf{u} f^{1 / 2} \Delta_{\mathbf{u}} f^{1 / 2} .
$$

The chains interact with each other in a way we need not know for the purposes of this section. If $f(\mathbf{u})$ may indeed be viewed as a correct "order parameter," the (extensive) free energy of interaction may be expressed in general as a functional expansion

$$
\begin{aligned}
F_{\text {int }}= & \frac{l}{P} \sum_{n=2}^{\infty} \int d \mathbf{u}_{1} d \mathbf{u}_{2} \cdots d \mathbf{u}_{n} \\
& \times K_{n}\left(\mathbf{u}_{1}, \mathbf{u}_{2}, \ldots, \mathbf{u}_{n}\right) f\left(\mathbf{u}_{1}\right) f\left(\mathbf{u}_{2}\right) \cdots f\left(\mathbf{u}_{n}\right) .
\end{aligned}
$$

To begin with, the reference state is isotropic $(f=1 / 4 \pi)$. A term of first order is absent because $K_{1}$ is a constant and the distribution is normalized to unity. The first term in Eq. (3.48) is a second virial term. In general, however, Eq. (3.48) need not be identified with a full virial series. The kernels $K_{n}$ are intensive variables signifying the complex interactions among the chains.

In order to compute the global persistence length, the test chain is hypothetically strained in an external field of the dipole type. ${ }^{45}$ The total free energy becomes

$$
F_{\text {tot }}[f(\mathbf{u})]=F_{\text {or }}+F_{\text {int }}-\frac{l h k_{B} T}{P} \int d \mathbf{u} \tau f(\mathbf{u})
$$

with $h$ a coupling constant. When we minimize the total free energy with respect to $f(\mathbf{u})$, bearing in mind the normalization of the latter, we obtain an integrodifferential equation which is self-consistent and nonlinear

$$
\begin{aligned}
-\frac{1}{2} f^{-1 / 2} \Delta_{\mathbf{u}} f^{1 / 2}= & E-\sum_{n=1}^{\infty} \int d \mathbf{u}, d \mathbf{u}_{2} \cdots d \mathbf{u}_{n}(n+1) \\
& \times K_{n+1}\left(\mathbf{u}_{1}, \mathbf{u}_{2}, \ldots, \mathbf{u}_{n}, \mathbf{u}\right) f\left(\mathbf{u}_{1}\right) \\
& \times f\left(\mathbf{u}_{2}\right), \ldots, f\left(\mathbf{u}_{n}\right)+h \tau .
\end{aligned}
$$

Here, $E$ is an eigenvalue. Without the external field $(h=0)$, we insist that Eq. (3.50) describes a nematic state. Various symmetry relations are thus required for the kernels $K_{n}$ and may easily be derived. Our model obviously does not account for the chirality of the DNA helix; the cholesteric pitch is assumed to be large enough. The global persistence length $P_{g}$ is now derived with the help of a linear response law ${ }^{45}$

$$
P_{g}=\left.\frac{1}{2} P \frac{\partial\langle\tau\rangle}{\partial h}\right|_{h=0} .
$$


Hence, we assume a regular perturbation solution for Eq. (3.50) exists for small $h$. As $h \rightarrow 0$, the distribution is written as $^{48}$

$$
\begin{aligned}
& f^{1 / 2}=f_{0}^{1 / 2}+h \Psi_{1}, \\
& \Psi_{1}(\tau)=-\Psi_{1}(-\tau)
\end{aligned}
$$

and so acquires a small antisymmetric component. For any $n$ we have

$$
\begin{aligned}
& \int d \mathbf{u}_{1} d \mathbf{u}_{2} \cdots d \mathbf{u}_{n} K_{n+1}\left(\mathbf{u}_{1}, \mathbf{u}_{2}, \ldots, \mathbf{u}_{n}, \mathbf{u}\right) \\
& \quad \times f_{0}\left(\mathbf{u}_{1}\right) f_{0}\left(\mathbf{u}_{2}\right) \cdots f_{0}\left(\mathbf{u}_{n-1}\right) f_{0}^{1 / 2}\left(\mathbf{u}_{n}\right) \Psi_{1}\left(\mathbf{u}_{n}\right)=0
\end{aligned}
$$

for the integrand is antisymmetric under the transformation $\left(\theta_{n}, \varphi_{n}\right) \rightarrow\left(\pi-\theta_{n}, \pi+\varphi_{n}\right)$. In effect, besides referring to a nematic state, $K_{n}$ is expected to be a complicated function of $\left|\sin \delta_{n}\right|$, where $\delta_{n}$ is the angle between an infinitesimal segment of orientation $\mathbf{u}_{n}$ and one of orientation $\mathbf{u}$. With this caveat, further analysis of Eq. (3.50) proceeds exactly along the lines of Vroege and Odijk ${ }^{48}$ to which the reader is referred. The global persistence length is given by

$$
P_{g}=2 \pi P \int_{0}^{1} d \tau_{2} \frac{\left[\int_{\tau_{2}}^{1} d \tau_{1} 2 \tau_{1} f_{0}\left(\tau_{1}\right)\right]^{2}}{\left(1-\tau_{2}^{2}\right) f_{0}\left(\tau_{2}\right)},
$$

which reduces to

$$
P_{g} \sim \frac{P}{2 \pi} \int_{0}^{1-\epsilon} d \tau \frac{1}{\left(1-\tau^{2}\right) f_{0}(\tau)}
$$

for highly oriented nematics. (The upper limit circumvents a divergence; deletion of a subdominant term is implied). In the Gaussian approximation [Eq. (3.3)], this becomes ${ }^{48}$

$$
P_{g} \sim \frac{\pi P e^{\alpha}}{2 \alpha^{2}} .
$$

Here, the parameter $\alpha$ has to be deduced from experiment, for instance by measuring the usual order parameter $\left(\alpha \simeq 3 /\left(1-S_{n}\right)\right)$. The free energy of a hairpin is essentially $\alpha k_{B} T$ to logarithmic order. The hairpin energy for the potential at high order [Eq. (3.2)], in the classical limit, was computed by de Gennes, ${ }^{46}$ and Williams and Warner. ${ }^{49}$ In the Gaussian limit, it becomes identical with a highly ordered self-consistent potential and equal to $\alpha k_{B} T$. Hence, the entropy of a hairpin bend must be very small.

\section{Statistical mechanics of a tight supercoil in a nematic}

The twist and writhe of a DNA supercoil are connected by White's relation. ${ }^{69,97}$ For a helical wormlike chain which is closed and unknotted, the torsional degrees of freedom in the partition function can be integrated out. The statistical analysis boils down to a consideration of a simple untwisted wormlike chain at a given writhe, ${ }^{30}$ a problem with a topological constraint. For flexible polymers, the statistical mechanics of a topologically restricted chain was initiated three decades ago. ${ }^{70,71}$ Edwards later presented a convenient functional representation for the partition function of a two- dimensional flexible coil wound several times around a point. $^{72}$ The problem is equivalent to the path integral of a charged quantum particle moving in a magnetic field. ${ }^{73,74} \mathrm{We}$ present a formal analysis for supercoiled DNA similar to the flexible-chain problem of Edwards. ${ }^{72}$

It is expedient to write the writhe $\mathrm{Wr}$ in a form advocated by le Bret ${ }^{4}$

$$
\mathrm{Wr}=-(2 \pi)^{-1} \int_{0}^{l} d s\left(\frac{d \varphi}{d s}\right) \cos \theta+\mathrm{Wr}_{z},
$$

where the unit vector $\mathbf{u}(s)$ is defined by Eq. (2.1) and $\mathrm{Wr}_{z}$ is the directional writhing number in the $z$ direction. For a tight supercoil in the semiclassical limit, undulations away from the classical or purely mechanical configuration are small, provided the latter is stable. Then, $\mathrm{Wr}_{z}$ is constant and fluctuations may be accounted for with the help of the integral in Eq. (3.57). ${ }^{30}$ Following Edwards' recipe ${ }^{72}$ we try to find a suitable "vector potential" $\mathbf{A}$ in terms of the polar angles [Eq. (2.1)],

$\mathbf{A}=\mathbf{A}(\mathbf{u}) \equiv(2 \pi)^{-1}(-\operatorname{cotg} \theta \sin \varphi, \operatorname{cotg} \theta \cos \varphi, 0)$

so that the writhe may be viewed as the "magnetic" interaction $^{73,74}$ of a "charged particle", with "velocity" $d \mathbf{u}$ / $d s$

$$
\Delta \mathrm{Wr} \equiv \mathrm{Wr}-\mathrm{Wr}_{z}=-\int_{0}^{l} d s \mathbf{A} \cdot \frac{d \mathbf{u}}{d s} .
$$

Hence, the pertinent partition function of the constrained semiflexible chain in a nematic field may be expressed by

$G\left(\mathrm{Wr} ; \mathbf{u}, l ; \mathbf{u}_{0}, 0\right)$

$$
\begin{aligned}
= & \int_{\mathbf{u}(0)=\mathbf{u}_{0}}^{\mathbf{u}(l)=\mathbf{u}} \mathscr{Q}[\mathbf{u}(s)] \delta\left(\mathbf{u}^{2}(s)-1\right) \\
& \times \delta\left(\Delta \mathrm{Wr}+\int_{0}^{l} d s \mathbf{A} \cdot \frac{d \mathbf{u}}{d s}\right) \exp -\left(U_{b}+U_{n}\right) / k_{B} T \\
= & \frac{1}{2 \pi} \int_{-\infty}^{\infty} d \mu e^{i \mu \Delta \mathrm{Wr}} G_{\mu}
\end{aligned}
$$

with

$$
\begin{aligned}
G_{\mu}= & \int_{\mathbf{u}(0)=\mathbf{u}_{0}}^{\mathbf{u}(l)=\mathbf{u}} \mathscr{Q}[\mathbf{u}(s)] \delta\left(\mathbf{u}^{2}(s)-1\right) \\
& \times \exp \left[-\frac{1}{2} P \int_{0}^{l} d s\left(\frac{d \mathbf{u}}{d s}\right)^{2}-\frac{1}{2 P} \int_{0}^{l} V(\mathbf{n} \cdot \mathbf{u}(s))\right. \\
& \left.+i \mu \int_{0}^{l} d s \mathbf{A} \cdot \frac{d \mathbf{u}}{d s}\right]
\end{aligned}
$$

This functional integral is now rewritten as the solution to the following differential equation: ${ }^{72,74}$

$$
\begin{aligned}
{\left[\frac{\partial}{\partial l}-\right.} & \left.\frac{1}{2 P}\left(\frac{\partial}{\partial \mathbf{u}}-i \mu \mathbf{A}(\mathbf{u})\right)^{2}+\frac{1}{2 P} V(\mathbf{u})\right] G_{\mu} \\
& =\delta(l) \delta\left(\mathbf{u}-\mathbf{u}_{0}\right) .
\end{aligned}
$$


Using the fact that $\mathbf{A}$ is divergence-free, we express this equation in terms of the polar angles

$$
\begin{aligned}
{\left[\frac{\partial}{\partial l}\right.} & \left.-\frac{1}{2 P}\left(\mathscr{L}_{\theta}+\frac{1}{\sin ^{2} \theta}\left(\frac{\partial}{\partial \varphi}-\frac{i \mu \cos \theta}{2 \pi}\right)^{2}-\Gamma \sin ^{2} \theta\right)\right]_{\mu} \\
& =\delta(l) \delta\left(\cos \theta-\cos \theta_{0}\right) \delta\left(\varphi-\varphi_{0}\right) .
\end{aligned}
$$

The partition function at constant writhe has been reduced to a form amenable to explicit analysis by the semiclassical method outlined in Sec. III B. Such a computation is obviously complicated; moreover, it is not easy to see how various terms have to be balanced in an approximation scheme. Equation (3.63) is left for now and supercoiling in a nematic field is addressed qualitatively in Sec. VII.

\section{QUALITATIVE CONCLUSIONS}

The conclusions of the preceding analyses are summarized in a qualitative scheme.

(1) A tight semicircular or circular bend with initial typical radius of curvature $R_{0}$ placed in a nematic field of strength $\Gamma$, retains its integrity and does not ripple when $\Gamma \lesssim P^{2} / R_{0}$. This criterion holds irrespective of the orientation of the plane of the bend with respect to the director. The singular case of a bend perpendicular to the director or nearly so, will be disregarded.

Undulations soften the loop. The free energy of entropic origin

$$
F_{\text {entr. }} \simeq-\frac{l}{P}
$$

associated with this softening is (a) extensive or proportional to the contour length of the bend; (b) independent of the nematic field. The orientational fluctuations expressing deviations from the classical path at positions $s$ and $t$ are well described by a Gaussian with second moment

$$
\left\langle\Delta \theta^{2}\right\rangle \simeq \frac{2|s-t|}{P}
$$

provided $s$ and $t$ are both not too close to the constrained ends.

(2) In the regime $\Gamma \gtrsim P^{2} / R_{0}$, the bend tightens $\left(R<R_{0}\right)$ and the rest of the chain is rippled on a characteristic length $\lambda=P / \alpha$. The resulting radius of curvature $R$ may be estimated from either of three equivalent statements.

(i) The relevant dimensionless group must be of order unity

$\Gamma R^{2} / P^{2} \simeq 1$

(ii) There is only one relevant scale in the complete problem $^{48}$

$\lambda=P / \alpha \simeq R$.

(iii) The orientational fluctuations within the tight bend must match with those in the rippled sections

$$
\left\langle\Delta \theta^{2}(\pi R)\right\rangle_{\text {bend }} \simeq\left\langle\theta^{2}\right\rangle_{\text {rip. }} \simeq \alpha^{-1} \text {. }
$$

An attempt is made to understand a variety of complicated problems qualitatively by applying Eqs. (4.1)-(4.5) in Secs. V to VIII.

\section{RING OF UNTWISTED DNA IN A NEMATIC}

\section{A. Closure of an unrippled ring}

Disregarding entropic fluctuations, Williams and Halperin $^{75}$ have discussed the cyclization of wormlike chains in nematic solvents. In another direction, Shimada and Yamakawa gave a quantitative description of ring formation for DNA with and without twist in the isotropic state. ${ }^{29}$ Their theory is of considerable use in interpreting the closure of DNA. $^{76,77}$ The analysis of Sec. III A provides a means for a simple interpolation between these two theories, at least for ring closure without twist.

The closure probability proposed has the form

$$
\begin{aligned}
J_{c} & \simeq 28 L^{-5} e^{-\mathscr{F}}, \\
\mathscr{F} & \simeq 7 L^{-1}-\frac{1}{2} L+\frac{1}{2} \Gamma L .
\end{aligned}
$$

In an isotropic solvent $(\Gamma=0)$, these two expressions are simply Eq. (75) of Ref. 29: The first term in Eq. (5.2) is a bending energy; the second is an extensive entropic term physically similar to the one computed in Sec. II, which describes the undulatory softening of the chain. When the nematic field is switched on $(\Gamma>0)$, the entropic term remains unaltered as argued in Sec. III A. The additional nematic energy is estimated assuming the closed chain is basically a circle. Thus the numerical coefficients of the first and third terms are not precise for general $\Gamma$ but the scaling structure of Eq. (5.2) is correct below the transition to a rippled state $\left(\Gamma L^{2} \lesssim 1\right)$.

The maximum probability of ring closure occurs when

$$
L_{m}=\frac{5-[25-14(1-\Gamma)]^{1 / 2}}{(1-\Gamma)} \text {. }
$$

In an isotropic solvent, $L_{m} \simeq 1.7$ as found by Shimada and Yamakawa. ${ }^{29}$ Note that $L_{m}$ is quite insensitive to the nematic field for $\Gamma=\mathscr{Q}(1)$, an effect arising from the entropy. In the regime $1 \ll \Gamma \ll L^{-2}$ for $L \ll 1$, entropy is unimportant and we regain the analysis by Williams and Halperin. ${ }^{75}$ In that case, the optimal radius $R_{m} \simeq \pi^{-1} P L_{m}$ is given by

$$
R_{m} \simeq \frac{P}{\Gamma^{1 / 2}} \simeq \frac{P}{\alpha} .
$$

Hence, $\mathscr{F}$ is essentially $\mathscr{O}\left(\Gamma^{1 / 2}\right)$. The prefactor in Eq. (5.1) is related to the decrease in orientational and translational entropy involved in forcing two DNA ends to meet. This should depend on $\Gamma$, but any $\Gamma$ dependence is reduced to minor logarithmic significance for $\Gamma>1$. Equations (5.1) and (5.2) are semiquantitatively correct in the entire unrippled regime $\left(L \lesssim 4, \Gamma L^{2} \lesssim 1\right)$.

\section{B. Ring in a strong nematic field}

In Sec. III B, we saw that the arm or two arms of a tight bend will ripple at sufficiently high fields. A closed circle of untwisted DNA ought to look like the elongated ring in Fig. 


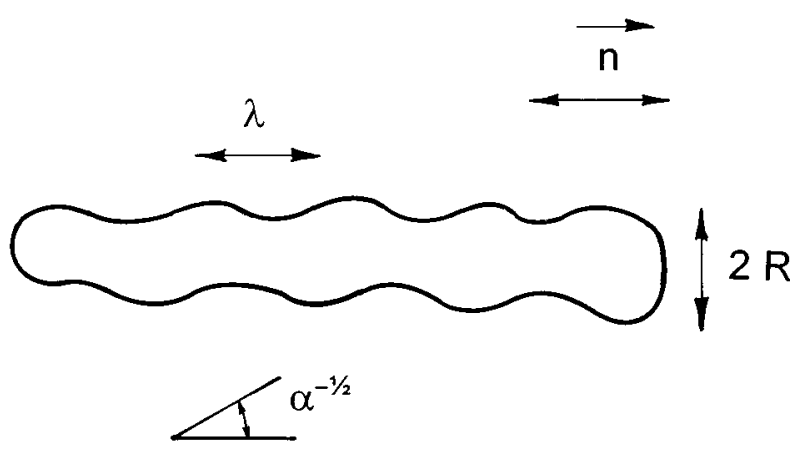

FIG. 7. An elongated ring in a strong nematic field.

7 in this case. The two tightly bent sections are approximated by semicircles of radius of curvature $R$. The two rippled sections have a total length $t=l-2 \pi R$ where $l$ is the contour length of the entire DNA chain which may be as long as one wishes but for the formation of hairpins. ${ }^{78}$ The total free energy of the chain consists of the following contributions.

(a) The two semicircles have a nematic energy

$$
F_{n} / k_{B} T \simeq \frac{1}{2} \pi \Gamma R P^{-1} \simeq \frac{1}{8} \pi \alpha^{2} R P^{-1}
$$

since in the rippling limit $\Gamma=\frac{1}{4} \alpha^{2}$.

(b) They have a bending energy

$$
F_{b} / k_{B} T \simeq \pi P R^{-1} \text {. }
$$

The entropy of Sec. III A is entirely negligible in the case when rippling occurs $(\Gamma \gg 1$; see below)

(c) The free energy of the rippled sections is

$$
\begin{aligned}
F_{\text {rip }} / k_{B} T & \simeq \Gamma^{1 / 2} t / P \\
& =\alpha t / 2 P .
\end{aligned}
$$

See Sec. VIII of Ref. 43. Note that it consists of two equal ${ }^{43}$ contributions, one coming from the orientational entropy and the other from the interaction of the DNA with the nematic field.

The total free energy is

$$
\begin{aligned}
F_{\text {tot }} / k_{B} T & =\left(F_{b}+F_{n}+F_{\text {rip }}\right) / k_{B} T \\
& =\frac{\pi P}{R}+\frac{\pi \alpha^{2} R}{8 P}+\frac{\alpha l}{2 P}-\frac{\pi \alpha R}{P} .
\end{aligned}
$$

The last term bearing a minus sign arises because the DNA has a finite size. The optimal radius of the semicircular sections is found by minimizing Eq. (5.8)

$$
R=\frac{P}{\alpha}\left(\frac{8}{1-8 \alpha^{-1}}\right)^{1 / 2} .
$$

This makes sense only if $\alpha>8$, a condition stemming from the finite length of the DNA. The chain is partly rippled only if $\alpha>8$ and if, in addition, $l>2 \pi R$. For $\alpha \leqslant 8$, the chain is simply a circle, to a first approximation, with

$$
F_{\text {tot }} / k_{B} T=\frac{\pi \alpha^{2} R}{8 P}+\frac{\pi P}{R},
$$

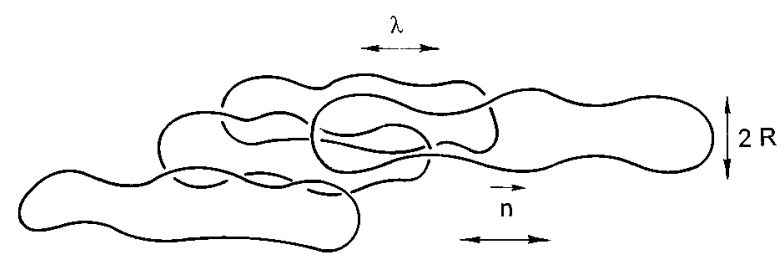

FIG. 8. Congested nematic rings forming a nematic state.

$$
R=\frac{8^{1 / 2} P}{\alpha}
$$

in agreement with Ref. 75. The latter equation bears a formal similarity to Eq. (5.4) in view of the unimportance of entropy for ring closure at high fields. The free energy of the elongated ring attains the form

$$
F_{\text {tot }} / k_{B} T \sim \frac{\alpha l}{2 P}+2^{-1 / 2} \pi \alpha
$$

at very high fields $(\alpha \gtrsim 20, \alpha l \gg P)$. In practice, there remains a substantial end correction arising from the two end hairpins.

\section{NEMATIC OF UNTWISTED DNA RINGS}

The preparation of nicked circular DNA or closed circular DNA without twist has become fairly routine. ${ }^{79}$ What happens if we pack untwisted DNA rings together in solution? A naive expectation would be to regard a ring as a disc, more or less. The disk diameter would be $2 R$ - the ring radius $R$ is less than $P$, say-and its diameter $d$, the (effective) diameter of the DNA helix. We might then formulate a criterion $^{80,81}$ for the onset to a nematic state

$$
\frac{R^{2} v_{*}}{d^{2}} \simeq 1
$$

where $v_{*}$ is a critical DNA volume fraction. It can be argued that this relation is probably valid despite the possibility of the rings deforming and rippling under the influence of the topological excluded-volume effect, which in turn depends on the orientational order.

Consider $N$ rings of contour length $l$ and diameter $d$ packed in an aqueous solution of volume $\mathscr{T}$. The DNA volume fraction is $v=(\pi / 4) l d^{2} N \mathscr{V}^{-1}$. The nematic state is defined by the Gaussian parameter $\alpha(\alpha \gg 1)$ and a second variational parameter $\eta$ is introduced,

$$
\eta \equiv \frac{2 \pi R}{l}
$$

for the rings possibly deforming under the nematic stress, into elongated rippled rings capped by approximately semicircular ends of radius $R$ (Fig. 8). The contour length is short enough to neglect hairpin formation. ${ }^{78}$ Now the rings cannot sample the complete configurational space in view of their topology. ${ }^{69,71}$ For instance, two test rings cannot be linked as in Fig. 9. In the isotropic state their topological excluded volume scales as $R^{3}$. For unelongated rings, this is modified 


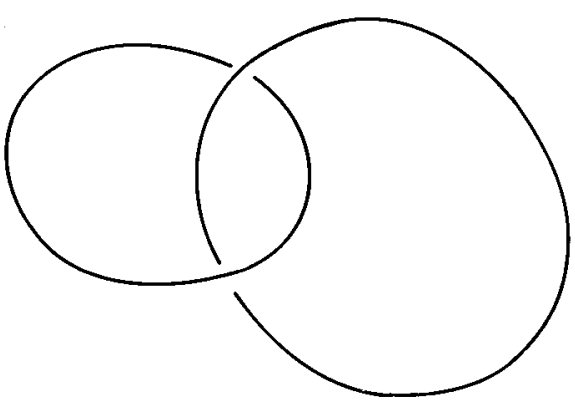

FIG. 9. A forbidden state of linked rings.

in the nematic by a factor $\alpha^{-1 / 2}$ because the orientational excluded volume scales, in the main, like $|\sin \gamma|$, where $\gamma$ is the angle between the normals to the planes of the two rings. We write the total free energy of a possible nematic in the second virial approximation as

$$
\begin{aligned}
F_{\text {tot }} / k_{B} T= & \frac{\left(l-2 \pi R+b_{1} R\right)^{2} R N^{2}}{\mathscr{T} \alpha^{1 / 2}}+\frac{(1-\eta)^{2} l^{2} d N^{2}}{\mathscr{T} \alpha^{1 / 2}} \\
& +\frac{R^{2} d N^{2}}{\mathscr{T} \alpha^{1 / 2}}+\frac{(1-\eta) l R d N^{2}}{\mathscr{V}}+\frac{(1-\eta) l \alpha N}{P} \\
& +\frac{P N}{R} .
\end{aligned}
$$

Here, most of the numerical coefficients have been deleted and the meaning of the terms is as follows:

(1) The first is the topological excluded volume where the elongation of the rings is taken into account (the coefficient $\left.b_{1}<2 \pi\right)$; (2) the second term represents the usual excluded volume between rippled strands of length $(1-\eta) l$; (3) the third term is the excluded volume between semicircles; (4) the fourth term signifies a cross interaction between the rippled strands and the semicircles; it is independent of $\alpha$ to a first approximation; (5) the fifth term represents the free energy of orientational confinement of the rippled strands; (6) the sixth term is the bending energy of the semicircles; the entropy of these may be safely neglected (Sec. IV).

Next, it is convenient to rewrite Eq. (6.3) in terms of a scaled volume fraction $\bar{v} \equiv P v / d$, a variable occurring in the theory of lyotropic polymer liquid crystals. ${ }^{63}$

$$
\begin{aligned}
\frac{P F_{\mathrm{tot}}}{N l k_{B} T} \simeq & \left(\frac{l}{d}\right) \bar{v} \alpha^{-1 / 2} \eta\left(1-b_{2} \eta\right)^{2}+\bar{v} \alpha^{-1 / 2}(1-\eta)^{2} \\
& +\bar{v} \alpha^{-1 / 2} \eta^{2}+\bar{v} \eta(1-\eta)+\alpha(1-\eta)+P^{2} / l^{2} \eta \\
\simeq & \left(\frac{l}{d}\right) \bar{v} \alpha^{-1 / 2} \eta\left(1-b_{2} \eta\right)^{2}+\alpha(1-\eta)+P^{2} / l^{2} \eta
\end{aligned}
$$

with a numerical coefficient $b_{2}<1$. At high salt, the aspect ratio $l / d$ is larger than about 30 , at least in practice, ${ }^{79}$ so it is a good idea to delete the three ordinary excluded-volume terms as the topological term is dominant. Order and elongation are strongly coupled in Eq. (6.4). The equilibrium state is found by minimizing $F_{\text {tot }}$ with respect to $\eta$ and $\alpha$.

$$
\begin{aligned}
& 2 \alpha^{3 / 2}=\left(\frac{l}{d}\right) \frac{\bar{v} \eta\left(1-b_{2} \eta\right)^{2}}{(1-\eta)}, \\
& \alpha^{3 / 2}=-\frac{P^{2} \alpha^{1 / 2}}{l^{2} \eta}+\left(\frac{l}{d}\right) \bar{v}\left(1-4 b_{2} \eta+3 b_{2} \eta^{2}\right) .
\end{aligned}
$$

We conclude the following from these expressions:

(i) The case $l \bar{v} / d=\mathscr{O}(1)$. If we let $l \lesssim 2 \pi P$, then a solution $\bar{v} \triangleq \bar{v}_{*}$ [Eq. (6.1)] is feasible with $\alpha=\mathscr{Q}(1)$ and $\eta \simeq \frac{1}{2}$. This is stretching the scaling analysis to its limit of validity. Accordingly, a naive disk picture seems not unreasonable at these very low volume fractions $\left(v \simeq 10^{-3}\right)$. But the rings are probably not completely stiff, but rippled a bit in the nematic phase coexisting with the isotropic at the transition.

(ii) The case $l \bar{v} / d \gg 1$. There appears to be a remote possibility of a solution to Eqs. (6.5) and (6.6) with $\alpha^{3 / 2}=\mathscr{Q}(\bar{v} / d)$ and $\eta \simeq \frac{1}{2}$, say, depending on the exact value of $b_{2}$. However, the free energy of this state would be substantially higher than that of the nematic defined by a second solution to these equations with $\eta \ll 1$, where the rings are markedly elongated

$$
\begin{gathered}
\eta^{5} \simeq \frac{P^{6} d^{2}}{l^{8} \bar{v}^{2}} \simeq \frac{P^{4} d^{4}}{l^{8} v}, \\
\alpha^{5 / 2} \simeq \frac{P^{2} \bar{v}}{l d} \simeq \frac{P^{3} v}{l d^{2}} .
\end{gathered}
$$

Note that in these expressions, topology, nematic order, bending, and orientational entropy are inextricably combined. The result is that the nematic order increases fairly weakly with the DNA volume fraction, and never becomes very high $(\alpha \lesssim 15)$.

Torbet and Di Capua ${ }^{82}$ have found that nicked circular pUC8 plasmids form a liquid-crystalline state at DNA volume fractions $v \ll 1$. The rings definitely become more elongated, the higher the DNA concentration. The liquid crystal state appears to be hexagonal so it may be presumptuous to compare their "radius" with the theoretical one, $R=\eta l / 2 \pi$, given by Eq. (6.7). Nevertheless, an identification of the experimental Bragg spacing $D_{B}$ with $R \sim v^{-2 / 5}$ is not devoid of interest: Torbet and Di Capua definitely rule out $D_{B} \sim v^{-1 / 2}$ which would hold if the slender DNA rings had fixed dimensions, but neither does their curve $D_{B} \sim v^{-1 / 3}$ seem a convincing fit to the data. A realistic exponent must lie in between $\frac{1}{2}$ and $\frac{1}{3}$. Therefore, there is a bit of evidence, albeit very tenuous, for the validity of the qualitative picture presented here. Note that even if the phase of Ref. 82 definitely turns out to be hexagonal, this does not preclude the possible and likely existence of nematic (cholesteric) states.

Theoretically, there are several issues that should be investigated. A biaxial phase may be possible for intermediately elongated rings. The feasibility of a hexagonal phase could be analyzed along the lines of recent theories. ${ }^{41,83}$ Furthermore, the long-range nematic interaction ${ }^{53}$ between the semicircular defects has been disregarded in the analysis above. 


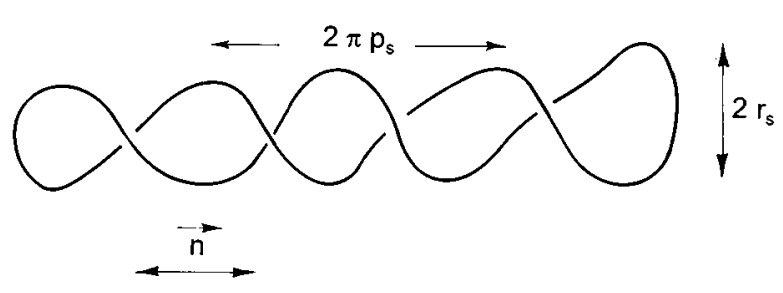

FIG. 10. Plectonemic supercoil in a nematic.

\section{PLECTONEMIC DNA IN A NEMATIC}

A purely qualitative analysis will be given of the uncoiling of plectonemic DNA by a nematic field. In reality, the long-range electrostatic repulsion between DNA segments couples strongly to entropy to give rise to undulationenhanced forces $^{83}$ [J. Ubbink and T. Odijk (manuscript in preparation)]. Marko and Siggia ${ }^{84}$ recently balanced entropy and repulsive forces in a theory of supercoils with and without tension. Still, it is of methodological interest to study the case where the diameter of the plectoneme is assumed constrained by a solely electrostatic exclusion ${ }^{11}$ without electric twist. ${ }^{85}$ Moreover, there is some evidence that the superhelical diameter is more or less constant in certain regimes. ${ }^{17,82}$ As seen in Sec. III D, a formal analysis of the entropy is complicated, but it will be dealt with approximately, after an introduction of the case without undulations.

We neglect end effects and branching: the slender plectonemic helix is supposed uniform with a diameter $2 r_{s}$ and pitch $2 \pi p_{s}$. The nematic torque is strong enough to align the superhelical axis along the director $\mathbf{n}$ of the nematic field (the $z$ direction Fig. 10). The total energy in an elastic approximation $^{86}$ is given by

$$
U_{\text {tot }} / l k_{B} T=\frac{1}{2} P R^{-2}+\frac{1}{2} C_{t} \Omega^{-2}+\frac{1}{2} \Gamma P^{-1}\left(1-(\mathbf{n} \cdot \mathbf{u})^{2}\right) .
$$

Here, $R$ is the radius of curvature and $l$ the total contour length of the DNA chain.

$$
R=\frac{r_{s}^{2}+p_{s}^{2}}{r_{s}}
$$

$C_{t}$ is a persistence length pertaining to twist and the local excess twist is defined with respect to the intrinsic twist $\omega_{0}$ of undisturbed DNA. The total molecular twist

$$
\mathrm{Tw}=\frac{1}{2 \pi} \int_{0}^{l} d s\left(\omega_{0}+\Omega\right)
$$

is connected with the writhe $\mathrm{Wr}$ and linking number Lk by White's relation $\mathrm{Lk}=\mathrm{Wr}+\mathrm{Tw}$. In general, the backbone helix of supercoiled DNA is twisted at a specific linking difference $^{79} \sigma=\left(\mathrm{Lk}-\mathrm{Lk}_{0}\right) / \mathrm{Lk}_{0}$ with $\mathrm{Lk}_{0}=l \omega_{0} / 2 \pi$. Hence, the local excess twist is given by

$$
\Omega=\omega_{0} \sigma-2 \pi l^{-1} \mathrm{Wr}
$$

for the supercoil may be assumed homogeneous, if end effects are neglected. For a left-handed helix, the writhe is ${ }^{6,87}$

$$
\mathrm{Wr}=\frac{p_{s} l}{2 \pi\left(r_{s}^{2}+p_{s}^{2}\right)} .
$$

For convenience, both $\sigma$ and $\mathrm{Wr}$ are here taken to be positive.

Inserting Eqs. (7.2), (7.4), and (7.5) into Eq. (7.1), we get the total energy for substantial superhelical pitch $\left(p_{s}>r_{s}\right)$, the case of interest if a nematic field is to exert some influence

$$
\begin{aligned}
U_{\text {tot }} / l k_{B} T= & \frac{P r_{s}^{2}}{2\left(r_{s}^{2}+p_{s}^{2}\right)^{2}}+\frac{1}{2} C_{t}\left(\omega_{0} \sigma-\frac{p_{s}}{\left(r_{s}^{2}+p_{s}^{2}\right)}\right)^{2} \\
& +\frac{\Gamma r_{s}^{2}}{2 P\left(r_{s}^{2}+p_{s}^{2}\right)} \simeq \frac{P r_{s}^{2}}{2 p_{s}^{4}}+\frac{1}{2} C_{t}\left(\omega_{0} \sigma-p_{s}^{-1}\right)^{2} \\
& +\frac{\Gamma r_{s}^{2}}{2 P p_{s}^{2}} .
\end{aligned}
$$

On minimizing $U_{\text {tot }}$ with respect to $p_{s}$, we find that the bending term is negligible in view of $C_{t}=\mathscr{Q}(P)$. The elongation factor is

$$
\begin{aligned}
& \frac{p_{s}}{p_{s, 0}} \simeq(1+\mathscr{N}), \\
& p_{s, 0}=1 / \omega_{0} \sigma, \\
& \mathscr{N} \equiv \frac{\Gamma r_{s}^{2}}{C_{t} P} .
\end{aligned}
$$

The nematic coupling parameter $\mathscr{N}$ may be of order unity in typical circumstances (e.g., $r_{s}=0.2 P=10 \mathrm{~nm} ; \omega_{0}=1.8$ $\left.\mathrm{nm}^{-1} ; \sigma=0.1 ; C_{t} \simeq P=50 \mathrm{~nm} ; \alpha \simeq 10 ; \Gamma=\alpha^{2} / 4=25\right)$. The parameter $\alpha$ has been measured recently in concentrated solutions of linear DNA, a persistence length long, ${ }^{68,88}$ and seems difficult to increase much beyond $\mathscr{Q}(10)$. At present, the degree of orientational order in suspensions of plectonemic DNA is unknown. ${ }^{82}$ On the whole, considerable nematic unwinding $(\mathscr{N} \gg 1)$ may be rare in the classical limit.

The effect of undulations is now investigated qualitatively in the case of weak and intermediate nematic fields in the sense of Sec. III A. At small pitch $\left(p_{s}<r_{s}\right)$, the plectoneme is basically a stack of almost parallel rings. A wind behaves typically like a fuzzy ring described in Sec. II with a small fuzzy thickness $\left(D \simeq R^{3 / 2} P^{-1 / 2} \ll R ; R \simeq r_{s} \ll P\right)$. Entropy, then, is important in the opposite limit $\left(p_{s}>r_{s}\right)$. In particular, we require the supercoil to be well defined with respect to the classical limit without undulations. The angle of the classical tangent vector with regard to the $z$ axis is about $r_{s} / p_{s}$. We insist that orientational fluctuations between adjacent winds be less than this number. These fluctuations conform to a Gaussian law (Sec. IV).

$$
\left(\frac{r_{s}}{p_{s}}\right)^{2} \gtrsim \frac{2 \pi p_{s}}{P} \text {. }
$$

This sets a fairly severe restriction on the value of $\Gamma$ via Eq. (7.7). Clearly, at a certain $\Gamma_{*}$, Eq. (7.6) may become dubious in view of the neglect of undulations. In Sec. III A, we concluded that the entropy of a tight bend is $k_{B} l / 4 P$, an exten- 


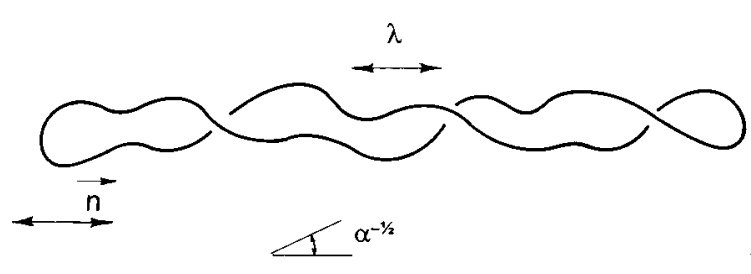

FIG. 11. Rippled supercoil in a strong nematic field.

sive term, so it should apply to a long tight plectoneme as well (A supercoil will have a certain winding number $M$ in Eq. (2.6). An exponential factor $\exp \frac{1}{4} L$ will again appear in the Green function analogous to the one given by Eq. (2.8). It is independent of the linking number and the nematic order, provided the radius of curvature is small enough $\left(\Gamma^{1 / 2} R<P\right)$, a condition often less stringent than Eq. (7.10). Hence, the invalidation of Eq. (7.10) signals a first possible breakdown of the classical limit with minor undulations.

Next, we go to the opposite extreme: a supercoil must ripple if the nematic field is strong enough (Fig. 11). From Sec. IV, we know the criterion for this to happen

$$
\alpha \gtrsim \frac{P}{R}=\frac{P r_{s}}{r_{s}^{2}+p_{s}^{2}} .
$$

In this limit, orientational order competes only with entropy. Note that the end effect discussed in Sec. V does not apply here, since the plectomene is very long. As soon as the supercoil becomes tangibly elongated by nematic stresses [Eq. (7.7)], there is a chance that it starts to ripple. A complete understanding of this process clearly requires a full quantitative analysis. Ultimately, the resulting rippled supercoil will still possess a writhe and twist depending on the nematic order.

In summary, it may be possible to unwind a plectonemic supercoil in an intermediate nematic field, with the entropic undulations playing only a minor role. At some stage, entropy does enter the picture and ultimately the DNA supercoil should ripple at a certain level of the nematic order. The calculations presented here, bear indirectly on the experiments by Torbet and Di Capua ${ }^{82}$ who prepared liquid crystals from plectonemic plasmid DNAs. For concentrations up to $80 \mathrm{mg} / \mathrm{ml}$, the supercoils seem to be arranged in hexagonal fashion and the superhelical pitch appears to be independent of the DNA concentration. Setting $r_{s}=8 \mathrm{~nm},{ }^{82} C_{t} \simeq 75 \mathrm{~nm}$ and $P=50 \mathrm{~nm}$, one would require a Gaussian parameter $\alpha \lesssim 5$ from Eq. (7.9), were we to tentatively assign a nematic torque exerted by the hexagonal packing. Such low values for $\alpha$ would be compatible with the low volume fractions $\bar{v}=P v / d=\mathscr{Q}(1)$. X-ray diffraction of fibers containing the superhelices seems to indicate that the supercoils are stretched at high concentrations ${ }^{82}(\vec{v} \gg 1$, purportedly implying $\alpha \gg 1$ ) but unfortunately the interpretation of the diffraction experiments is not unambiguous. Yevdokimov et al. ${ }^{89}$ have also investigated liquid crystalline phases of various types of superhelical DNA. They express uncertainty as to the precise nature of their phases (cholesteric, nematic or hexagonal), an issue that evidently warrants further study.
Finally, Marko and Siggia have published certain conclusions concerning plectonemes and solenoids under tension, ${ }^{36,84}$ a problem which is of course related to the one discussed here. In their work, ${ }^{84}$ there may be a regime at low ionic strength where $r_{s}$ is constrained by strong electrostatics, and $p_{s}$ is quite large, which apparently corresponds to the classical limit discussed here. Without doubt, the problem of undulations (and the hexagonal phase) will have to be reinvestigated within a more complete undulation theory. ${ }^{83}$

\section{PITCH OF CHOLESTERIC SUPERCOILS}

\section{A. Tight supercoils}

Tight plectonemic DNA forms a liquid crystal because the superhelices are stiff. In a regime dominated by entropy, the superhelical persistence length $P_{s}$ may be estimated by using the angular fluctuations of one wind (see Secs. IV and VII)

$$
\begin{aligned}
& \left\langle\Delta \theta^{2}\right\rangle \simeq \frac{2 \pi\left(r_{s}^{2}+p_{s}^{2}\right)^{1 / 2}}{P} \\
& P_{s} \simeq \frac{2 \pi p_{s}}{\left\langle\Delta \theta^{2}\right\rangle} \simeq \frac{2 p_{s} P}{\left(r_{s}^{2}+p_{s}^{2}\right)^{1 / 2}} .
\end{aligned}
$$

The numerical coefficient 2 has been added since $P_{s}$ must be twice the backbone persistence length for two chains at zero writhe. Eq. (7.2) is definitely an underestimate judging from computer simulations ${ }^{90}$ and experiments ${ }^{17,20}$ on tightly interwound DNA. This is not difficult to understand since the fluctuations are actually smaller than the ideal undulations inherent in Eq. (8.1), in view of interchain repulsion within the superhelix. Marko and Siggia have attempted to model this effect by a harsh power law ${ }^{14}$ and recently by electrostatics without undulation enhancement. ${ }^{84}$ The electrostatic repulsion between DNA chains decays exponentially and exerts a twist ${ }^{85}$ we expect undulation enhancement of the kind formulated in Ref. 83 to occur within the superhelix, a topic we are currently addressing [J. Ubbink and T. Odijk (manuscript in preparation)].

In a liquid crystal of plectonemes, the DNA superhelices may act like rods ${ }^{80}$ or super-wormlike chains ${ }^{91}$ depending on the value of the deflection length ${ }^{63} \lambda_{s}=P_{s} / \alpha$; the value of $P_{s}$ has to be estimated from simulation or experiment, for now. Next, it is evidently feasible to set up conditions such that the superhelical threads are neither perturbed nor rippled by the nematic field, whatever its origin, at least to a first approximation [see Eq. (7.9)]. This is the case we focus on here for a discussion of the cholesteric phase.

\section{The superhelices are effective rods $\left(\frac{1}{2} / \leqslant \lambda_{s}\right)$}

In the virial theory ${ }^{92,93}$ of the pitch of a cholesteric suspension of chiral rods, there is a vector kernel $\mathbf{C}_{c}\left(\mathbf{u}_{1}, \mathbf{u}_{2}\right)$ for two test rods of orientations $\mathbf{u}_{1}$ and $\mathbf{u}_{2}$, within the virial term of first order. For two supercoiled threads, the following form is proposed:

$$
\mathbf{C}_{c}\left(\mathbf{u}_{1}, \mathbf{u}_{2}\right)=j\left(r_{s} \mathrm{Wr} / l\right) d l^{2} r_{s}\left(\mathbf{u}_{1} \times \mathbf{u}_{2}\right)\left(\mathbf{u}_{1} \cdot \mathbf{u}_{2}\right) .
$$

This is plausibly argued as follows: It is proportional to ${ }^{92,93}$ 
(i) the excluded volume $\sim l^{2} r_{s}$ between two supercoils of length $\frac{1}{2} l$ and diameter $2 r_{s}$.

(ii) The thickness of the superhelical threads, i.e., the distance between the two DNA chains.

(iii) The orientational part $\mathbf{u}_{1} \times \mathbf{u}_{2}$ of the excluded volume.

(iv) The angular factor $\mathbf{u}_{1} \cdot \mathbf{u}_{2}$, for $\mathbf{C}_{c}$ is zero in the crossed configuration; note that $\mathbf{C}_{c}\left(\mathbf{u}_{1}, \mathbf{u}_{2}\right)=-\mathbf{C}_{c}\left(\mathbf{u}_{2}, \mathbf{u}_{1}\right)$ and $\mathbf{C}_{c}\left(\mathbf{u}_{1}, \mathbf{u}_{2}\right)=\mathbf{C}_{c}\left(\mathbf{u}_{1}-\mathbf{u}_{2}\right)=\mathbf{C}_{c}\left(-\mathbf{u}_{1}, \mathbf{u}_{2}\right)$.

(v) A dimensionless function $j$ depending on the superhelical chirality $r_{s} \mathrm{Wr} / l$. The total contour length of the DNA is $l$ and the length of a plectoneme is about $\frac{1}{2} l$. As the writhe tends to zero, $j$ goes to zero.

Equation (8.3) leads to a pitch $^{93} \mathscr{P}$ of the cholesteric phase of rodlike supercoils

$$
\begin{aligned}
& \mathscr{P} \simeq \frac{d l}{r_{s} v\left|j\left(r_{s} \mathrm{Wr} / l\right)\right|}, \\
& \alpha \simeq\left(l v / r_{s}\right)^{2},
\end{aligned}
$$

where $v$ is the DNA volume fraction.

\section{Semiflexible plectonemes $\left(\frac{1}{2} l \geq \lambda_{s}\right)$}

The supercoiled threads are semiflexible, and statistically speaking, may be viewed as a sequence of deflection segments of length $\lambda_{s}$ (see Ref. 63). As in Ref. 93, $\frac{1}{2} l$ in Eqs. (8.4) and (8.5) should be replaced by $\lambda_{s}=P_{s} / \alpha$ (but note that $\mathrm{Wr} \sim l)$.

$$
\begin{aligned}
& \mathscr{P} \simeq \frac{d^{7 / 3} P_{s}^{1 / 3}}{r_{s}^{5 / 3} v^{5 / 3} \mid j\left(r_{s} \mathrm{Wr} / l\right)}, \\
& \alpha \simeq\left(P_{s} r_{s} v / d^{2}\right)^{2 / 3} .
\end{aligned}
$$

Equation (8.6) predicts a fairly low pitch for the cholesteric phase, at least if the writhe is not too small. The chirality of the DNA helix itself has been disregarded in Eq. (8.3). This may be reasonable, for the global superhelical chirality may be thought to dominate when two plectonemes collide. Here, we have neglected branching and end effects. Nevertheless, note that in the semiflexible case, all branches will align along the director in view of the strong cholesteric torque, so the liquid crystal is a suspension of deflection segments and our scaling analysis may remain valid.

\section{B. Loose supercoils}

A cholesteric suspension of DNA supercoils that are nontight—rippled or not—has a pitch because of three effects: (i) the DNA double helix itself is chiral; (ii) the supercoils are chiral objects interacting with each other; (iii) the average interaction between the segments of one supercoil is chiral. For loose supercoils, the first effect is very similar to that involved in a suspension of linear DNA molecules. Straley's theory ${ }^{92}$ as modified by the author, ${ }^{93}$ might be useful here although only at fairly low volume fractions. At high concentrations, DNA approaches the hexagonal phase so the pitch becomes anomalous. ${ }^{94,95}$ One recent theory quantifies the competition between braiding and chirality for closepacked chiral macromolecules. ${ }^{96}$ Here, we leave these problems aside and concentrate on the third type of chiral inter-

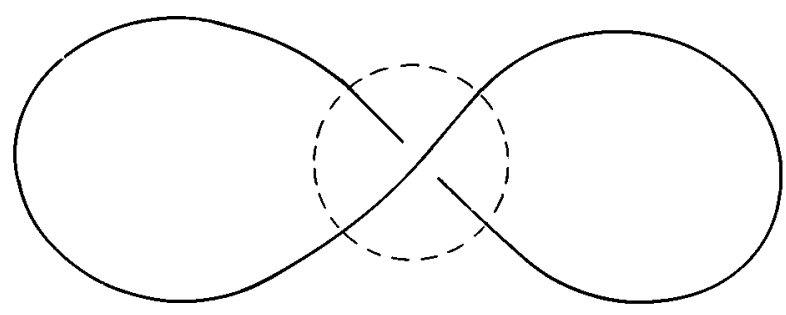

FIG. 12. A loose supercoil displaying a chiral excluded-volume effect.

action which seems to bear on recent experimental work. ${ }^{55} \mathrm{~A}$ scaling relation is given between the cholesteric pitch and the writhe, a measure of the handedness of a supercoil. For loose supercoils, the liquid crystal is a solution of interacting deflection segments so the qualitative analyses below should hold even if the supercoils branch.

Consider, first, one long supercoil of backbone contour length $l$ and helix diameter $d$ in a nematic medium whose properties will be discussed below. The supercoil is viewed as a sequence of $l / \epsilon$ slender rodlets, each of length $\epsilon(\epsilon \gg d)$. The DNA helix must be straight, or approximately so, on the scale of $\epsilon$. The achiral rodlets interact with each other via an excluded-volume effect. But the average interaction is chiral for it is topological, because the rodlets are connected into one supercoil (Fig. 12). Following previous work, ${ }^{92,93}$ we can write the increase in the free energy of chiral interaction as

$$
\begin{aligned}
F_{\mathrm{chi}} \simeq & k_{B} T \sum_{i \neq j} \sum_{i j}\left\langle\mathscr{V}_{i j}^{-1} \iint d \mathbf{u}_{i} d \mathbf{u}_{j} f\left(\mathbf{n} \cdot \mathbf{u}_{i}\right)\right. \\
& \left.\times \mathbf{C}_{c}\left(\mathbf{u}_{i}, \mathbf{u}_{j}\right) \cdot \nabla f\left(\mathbf{n} \cdot \mathbf{u}_{j}\right)\right\rangle .
\end{aligned}
$$

Here, $\mathbf{n}$ is the director which reflects some cholesteric organization owing to the perturbative presence of the supercoil, $f$ is the orientation distribution depending implicitly on position $\mathbf{r}$ via $\mathbf{n}(\mathbf{r}), \mathbf{u}_{i}$ and $\mathbf{u}_{j}$ are the unit vectors pointing along rodlets $i$ and $j$, and $\mathscr{V}_{i j}$ is the characteristic volume sampled by rodlet $i$ if we keep $j$ fixed. The average is over all configurations of the supercoil. The vector $\mathbf{C}_{c}$ was defined earlier [Eq. (8.3)] but is now formally defined ${ }^{92}$ in terms of a Mayer function $\Phi\left(\mathbf{u}_{i}, \mathbf{u}_{j}, \boldsymbol{\xi}_{i j}\right)$ where $\boldsymbol{\xi}_{i j}$ is the vector between the centers of the rodlets $i$ and $j$

$$
\mathbf{C}_{c}\left(\mathbf{u}_{i}, \mathbf{u}_{j}\right)=-\int d \boldsymbol{\xi}_{i j} \boldsymbol{\xi}_{i j} \Phi\left(\mathbf{u}_{i}, \mathbf{u}_{j}, \boldsymbol{\xi}_{i j}\right)
$$

For overlapping rodlets, we approximate the Mayer function by the form

$$
\Phi\left(\mathbf{u}_{i}, \mathbf{u}_{j}, \boldsymbol{\xi}_{i j}\right)=\Phi\left(\mathbf{u}_{j}, \mathbf{u}_{i},-\boldsymbol{\xi}_{i j}\right) \simeq \xi_{i j}^{-1} \mathbf{u}_{i} \times \mathbf{u}_{j} \cdot \boldsymbol{\xi}_{i j}\left(\mathbf{u}_{i} \cdot \mathbf{u}_{j}\right)
$$

and zero otherwise. This would be compatible with the vector $\mathbf{C}_{c}$ for screws chosen earlier by Straley. ${ }^{92}$

Next, we compute the chiral free energy in the continuum limit, adopting a scaling Ansatz $\xi_{i j}^{3} \simeq \mathscr{V}_{i j}$ 


$$
\begin{aligned}
F_{\mathrm{chi}} \simeq & k_{B} T \boldsymbol{\epsilon}^{-2} \int_{0}^{l} d s_{1} \int_{0}^{l} d s_{2} \int d \mathbf{u}\left(s_{1}\right) \int d \mathbf{u}\left(s_{2}\right) \int d \boldsymbol{\xi}_{i j} \\
& \times\left\langle\left(\frac{\mathbf{u}\left(s_{1}\right) \times \mathbf{u}\left(s_{2}\right) \cdot \boldsymbol{\xi}_{12}}{\xi_{12}^{3}}\right) f\left(\mathbf{n} \cdot \mathbf{u}\left(s_{1}\right)\right) \frac{\boldsymbol{\xi}_{12}}{\xi_{12}}\right. \\
& \left.\cdot \nabla f\left(\mathbf{n} \cdot \mathbf{u}\left(s_{2}\right)\right)\right\rangle .
\end{aligned}
$$

To zero order, $\left\langle\mathbf{u}_{i} \cdot \mathbf{u}_{j}\right\rangle \simeq 1$, by way of the generally high order in lyotropic systems. Introducing a further preaveraging approximation consistent with a scaling analysis, we now integrate only one part of the integrand over $s_{1}$ and $s_{2}$ in order to extract a writhing number ${ }^{69,97}$ from Eq. (8.11)

$$
\mathrm{Wr} \equiv \frac{1}{4 \pi} \int_{0}^{l} d s_{1} \int_{0}^{l} d s_{2} \frac{\mathbf{u}\left(s_{1}\right) \times \mathbf{u}\left(s_{2}\right) \cdot \boldsymbol{\xi}_{12}}{\xi_{12}^{3}} .
$$

The integral over $\boldsymbol{\xi}_{12}$ for overlapping rodlets yields an excluded volume contribution $\epsilon^{2} d$. Accordingly, Eq. (8.11) reduces to

$$
F_{\mathrm{chi}} \simeq k_{B} T d|\langle\mathrm{Wr}\rangle| \int d \mathbf{u}_{1} \int d \mathbf{u}_{2} f\left(\mathbf{n} \cdot \mathbf{u}_{1}\right)\left|\nabla f\left(\mathbf{n} \cdot \mathbf{u}_{2}\right)\right|,
$$

which is independent of the rodlet size $\epsilon$.

We are finally in a position to calculate the pitch $\mathscr{P}$. For a highly ordered cholesteric, we have ${ }^{93}|\boldsymbol{\nabla} f| \simeq \alpha^{1 / 2}|\mathbf{n} \cdot \boldsymbol{\nabla} \times \mathbf{n}|$. Let there be $N$ supercoils enclosed in a volume $\mathscr{T}$. Then the free energy density is

$$
F_{\mathrm{chi}} / \mathscr{T} \simeq k_{B} T d N \mathscr{T}^{-1} \alpha^{1 / 2}|\langle\mathrm{Wr}\rangle||\mathbf{n} \cdot \nabla \times \mathbf{n}| .
$$

Hence, the pitch is given in terms of the DNA volume fraction

$$
\mathscr{P} \simeq \frac{K_{\mathrm{tw}}|\mathbf{n} \cdot \boldsymbol{\nabla} \times \mathbf{n}| \mathscr{V}}{F_{\mathrm{chi}}} \simeq \frac{K_{\mathrm{tw}} l d}{k_{B} T v \alpha^{1 / 2}|\langle\mathrm{Wr}\rangle|} .
$$

A relation between the pitch and the twist elastic constant $K_{\mathrm{tw}}$ has been used. (A large factor of $2 \pi$ has been deleted.)

It is possible to simplify Eq. (8.15) a bit further by supposing the supercoils are in a more or less rippled state (see Sec. VII). The orientational order is then determined mainly by the interactions between DNA segments. In that case, we may use the approximation ${ }^{37,40}$

$$
K_{\mathrm{tw}} \simeq \frac{k_{B} T \alpha^{1 / 2}}{d}
$$

so the pitch may be expressed in a seductively simple fashion

$$
\mathscr{P} \simeq \frac{l}{v|\langle\mathrm{Wr}\rangle|} .
$$

This seems quite plausible after the fact. For very long chains, one expects $\langle\mathrm{Wr}\rangle \simeq \Delta \mathrm{Lk}$ even in a cholesteric phase. Hence, we would have a useful relation between the pitch and the specific linking difference

$$
\mathscr{P} \simeq \frac{h_{0}}{v|\sigma|}
$$

with $h_{0}=2 \pi / \omega_{0}=3.4 \mathrm{~nm}$. If there is a contribution from the intrinsic chirality of the DNA itself, which would lead to a pitch $\mathscr{P}_{\text {int }}$, then the total pitch would be

$$
\mathscr{P}_{\text {tot }}=\left|\frac{\mathscr{P} \mathscr{P}_{\text {int }}}{\mathscr{P} \pm \mathscr{P}_{\text {int }}}\right| \text {. }
$$

The choice of the sign depends on the handedness of the respective interactions.

The excluded volume effect for DNA is influenced by screened electrostatics so we have to deal, provisionally at least, with an effective diameter $d_{\text {eff }}$. Considerations concerning the twist modulus ${ }^{98}$ imply that $d$ in Eq. (8.15) be replaced by $d^{2} d_{\text {eff }}^{-1}$, and $d$ in Eq. (8.16) be replaced by $d_{\text {eff }}$. The pitch is modified to

$$
\mathscr{P} \simeq \frac{l d^{2}}{d_{\mathrm{eff}}^{2} v|\langle\mathrm{Wr}\rangle|} .
$$

It is stressed that the expressions for the pitch are merely scaling estimates in view of the rather severe approximations employed. There is some recent evidence of considerable interest for a direct relation between supercoiling and cholesteric organization. Reichl et al. ${ }^{55}$ have found that within bacteria, congested interwound supercoils of plasmid DNA form cholesteric phases. The circular dichroism spectra were a sensitive monitor of the superhelical density of the DNA. In one measurement at a volume fraction $v \simeq 0.015$ and a specific linking difference $\sigma \simeq-0.06$, they determined a pitch $\mathscr{P} \simeq 6 \mu \mathrm{m}$ which compares favorably with the rough estimate of $4 \mu \mathrm{m}$ given by Eq. (7.18). It is hoped that the pitch will be measured as a function of $\sigma$ in future experiments.

\section{CONCLUDING REMARKS}

The main purpose of this work has been to try to grasp, in a qualitative way, the behavior of congested DNA in problems of biophysical interest. A fairly severe drawback of our computations is the cavalier attitude toward the interactions between the DNA molecules. This is especially true in Sec. VII. We hope to come back to the issue of electrostatic interactions in the near future. The principal focus here has been the often intricate relation between entropy, order, curvature, and topology. The analysis of Secs. V to VIII is strictly qualitative, numerical coefficients not always of order unity, having been deleted either for the sake of simplicity or because they are unknown. A complete quantitative treatment of congested supercoils seems no mean task as does an implementation of Monte Carlo simulations of such suspensions given the current status of simulated nematic worms. $^{99}$

For tight DNA curves, entropy appears to be important mostly in closure problems as discussed in Sec. V A. Entropy does play a major role when the external field is strong, causing the DNA chain to ripple; but then the rippled sections undulate along the director and so are bent only weakly. However, the possibility is left open that undulations may well be frozen out for curved DNA in a strong field with positional order as occurs in a nucleosome particle. ${ }^{35}$ 


\section{ACKNOWLEDGMENTS}

I thank A. Duyndam, J. F. Marko, R. de Vries, and J. Ubbink for discussions.

${ }^{1}$ L. D. Landau and E. M. Lifshitz, Statistical Physics, 3rd ed. (Pergamon, Oxford, 1980), Part 1

${ }^{2}$ C. J. Benham, Proc. Natl. Acad. Sci. USA 74, 2397 (1977).

${ }^{3}$ C. J. Benham, Biopolymers 18, 609 (1979).

${ }^{4}$ M. Le Bret, Biopolymers 18, 1709 (1979).

${ }^{5}$ M. Le Bret, Biopolymers 23, 1835 (1984).

${ }^{6}$ J. H. White and W. R. Bauer, J. Mol. Biol. 189, 329 (1986).

${ }^{7}$ M. Hao and W. K. Olson, Macromolecules 22, 3292 (1989).

${ }^{8}$ T. Schlick and W. K. Olson, J. Mol. Biol. 223, 1089 (1992).

${ }^{9}$ I. Tobias and W. K. Olson, Biopolymers 33, 639 (1993).

${ }^{10}$ M. O. Fenley, W. K. Olson, I. Tobias, and G. S. Manning, Biophys. Chem. 50, 255 (1994).

${ }^{11}$ N. G. Hunt and J. E. Hearst, J. Chem. Phys. 95, 9329 (1991).

${ }^{12}$ Y. Shi and J. E. Hearst, J. Chem. Phys. 101, 5186 (1994).

${ }^{13}$ F. Jülicher, Phys. Rev. E 49, 2429 (1994).

${ }^{14}$ J. F. Marko and E. D. Siggia, Macromolecules 27, 981 (1994).

${ }^{15}$ D. Shore, J. Langowski, and R. L. Baldwin, Proc. Natl. Acad. Sci. USA 78, 4833 (1981).

${ }^{16}$ D. Shore and R. L. Baldwin, J. Mol. Biol. 170, 957 (1983).

${ }^{17}$ T. C. Boles, J. H. White, and N. R. Cozzarelli, J. Mol. Biol. 213, 931 (1990).

${ }^{18}$ W. H. Taylor and P. J. Hagerman, J. Mol. Biol. 212, 363 (1990).

${ }^{19}$ M. Adrian, B. ten Heggeler-Bordier, W. Wahli, A. Z. Stasiak, A. Stasiak, and J. Dubochet, EMBO J. 9, 4551 (1990).

${ }^{20}$ J. Bednar, P. Furrer, A. Stasiak, J. Dubochet, E. H. Egelman, and A. D. Bates, J. Mol. Biol. 235, 825 (1994).

${ }^{21}$ A. V. Vologodskii, V. V. Anshelevich, A. V. Lukashin, and M. D. FrankKamenetskii, Nature (London) 280, 294 (1979).

${ }^{22}$ K. V. Klenin, A. V. Vologodskii, V. V. Anshelevich, A. M. Dykhne, and M. D. Frank-Kamenetskii, J. Biol. Struct. Dyn. 5, 1173 (1988).

${ }^{23}$ K. V. Klenin, A. V. Vologodskii, V. V. Anshelevich, A. M. Dykhne, and M. N. Frank-Kamenetskii, J. Mol. Biol. 217, 413 (1991).

${ }^{24}$ A. V. Vologodskii, S. D. Levene, K. V. Klenin, M. N. Frank-Kamenetskii, and N. R. Cozzarelli, J. Mol. Biol. 227, 1224 (1992).

${ }^{25}$ J. Shimada and H. Yamakawa, Biopolymers 27, 657 (1988).

${ }^{26}$ C. J. Camacho, M. E. Fisher and R. R. P. Singh, J. Chem. Phys. 94, 5693 (1991).

${ }^{27}$ M. C. Tesi, E. J. Janse van Rensburg, E. Orlandini., D. W. Summers, and S. G. Whittington, Phys. Rev. E 49, 868 (1994).

${ }^{28}$ H. Yamakawa and W. H. Stockmayer, J. Chem. Phys. 57, 2843 (1972).

${ }^{29}$ J. Shimada and H. Yamakawa, Macromolecules 17, 689 (1984).

${ }^{30}$ J. Shimada and H. Yamakawa, J. Mol. Biol. 184, 319 (1985).

${ }^{31}$ C. J. Benham, Phys. Rev. A 39, 2582 (1989).

${ }^{32}$ E. Guitter and S. Leibler, Europhys. Lett. 17, 643 (1992).

${ }^{33}$ J. E. Hearst and N. G. Hunt, J. Chem. Phys. 95, 9322 (1991).

${ }^{34}$ G. S. Manning, J. Chem. Phys. 96, 4007 (1992).

${ }^{35} \mathrm{~T}$. Odijk, Macromolecules 26, 6897 (1993).

${ }^{36}$ J. F. Marko and E. D. Siggia, Science 265, 506 (1994)

${ }^{37}$ A. Yu. Grosberg and A. V. Zhestkov, Polym. Sci. USSR 28, 97 (1986).

${ }^{38}$ A. Yu. Grosberg and A. V. Zhestkov, J. Biomol. Struct. Dyn. 3, 859 (1986).

${ }^{39}$ A. Yu. Grosberg and A. V. Zhestkov, Biofizika 30, 233 (1986).

${ }^{40}$ T. Odijk, Liq. Cryst. 1, 553 (1986).

${ }^{41}$ J. V. Selinger and R. F. Bruinsma, Phys. Rev. A 43, 2910 (1991).

${ }^{42}$ J. Ubbink and T. Odijk, Biophys. J. 68, 54 (1995).

${ }^{43}$ T. Odijk, Macromolecules 19, 2313 (1986).

${ }^{44}$ M. H. Li, A. Brûlet, J. P. Cotton, P. Davidson, C. Strazielle, and P. Keller, J. Phys. II 4, 1843 (1994).

${ }^{45}$ A. R. Khokhlov and A. N. Semenov, J. Phys. A 15, 1361 (1982).

${ }^{46}$ P. G. de Gennes, in Polymer Liquid Crystals, edited by A. Ciferri, W. R. Krigbaum, and R. B. Meyer (Academic, New York, 1982).

${ }^{47}$ M. Warner, J. M. F. Gunn, and A. Baumgärtner, J. Phys. A 18, 3007 (1985).

${ }^{48}$ G. J. Vroege and T. Odijk, Macromolecules 21, 2848 (1988).

${ }^{49}$ D. R. M. Williams and M. Warner, J. Phys. 51, 317 (1990).

${ }^{50}$ M. Warner and D. R. M. Williams, J. Phys. II 2, 471 (1991).

${ }^{51}$ D. R. M. Williams, J. Phys. A 24, 4427 (1991).
${ }^{52}$ D. R. M. Williams and A. Halperin, Macromolecules 26, 2025 (1993).

${ }^{53}$ J. V. Selinger and R. F. Bruinsma, J. Phys. II 2, 1215 (1992).

${ }^{54}$ R. D. Kamien, P. Le Doussal, and D. R. Nelson, Phys. Rev. A 45, 8727 (1992).

${ }^{55}$ Z. Reich, E. J. Wachtel, and A. Minsky, Science 264, 1460 (1994).

${ }^{56} \mathrm{H}$. Yamakawa, Modern Theory of Polymer Solutions (Harper and Row, New York, 1971).

${ }^{57}$ M. V. Berry and K. E. Mount, Rep. Prog. Phys. 35, 315 (1972).

${ }^{58}$ R. E. Langer, Phys. Rev. 51, 669 (1937).

${ }^{59}$ This uniform validity is not accidental. An approximation for $P_{n}$ uniformly valid down to $\theta=0$ is a Bessel function (Ref. 57). The integration can now be performed exactly and, as it turns out, $G$ is now also given by Eq. (2.10) (I thank A. Duyndam for pointing this out to me). The asymptotic analysis [Eqs. (2.6)-(2.9)] illustrates the line of argumentation useful in the general case.

${ }^{60}$ M. C. Gutzwiller, J. Math. Phys. 8, 1979 (1967)

${ }^{61}$ M. C. Gutzwiller, J. Math. Phys. 10, 1004 (1969).

${ }^{62}$ M. C. Gutzwiller, Chaos in Classical and Quantum Mechanics (Springer, New York, 1990)

${ }^{63}$ T. Odijk, Macromolecules 19, 2313 (1986).

${ }^{64}$ L. D. Landau and E. M. Lifshitz, Quantum Mechanics, 2nd ed. (Pergamon, Oxford, 1965)

${ }^{65} \mathrm{H}$. T. Davis, Introduction to Nonlinear Differential and Integral Equations (Dover, New York, 1962).

${ }^{66}$ T. E. Strzelecka and R. L. Rill, Macromolecules 24, 5124 (1991).

${ }^{67}$ K. Merchant and R. L. Rill, Macromolecules 27, 2365 (1994).

${ }^{68}$ K. Kassapidou, R. K. Heenan, W. Jesse, M. E. Kuil, and J. R. C. van der Maarel, Macromolecules 28, 3230 (1995).

${ }^{69}$ M. D. Frank-Kamenetskii and A. V. Vologodskii, Sov. Phys. Usp. 24, 697 (1982).

${ }^{70}$ H. L. Frisch and S. Prager, J. Chem. Phys. 46, 1475 (1967).

${ }^{71}$ S. F. Edwards, Proc. Phys. Soc. 91, 513 (1967).

${ }^{72}$ S. F. Edwards, in Molecular Fluids, edited by R. Balian and G. Weill (Gordon and Breach, London, 1976).

${ }^{73}$ R. P. Feynman, Rev. Mod. Phys. 20, 367 (1948).

${ }^{74}$ L. S. Schulman, Techniques and Applications of Path Integration (Wiley, New York, 1981).

${ }^{75}$ D. R. M. Williams and A. Halperin, J. Phys. (Paris) II 3, 69 (1993).

${ }^{76}$ D. Shore and R. L. Baldwin, J. Mol. Biol. 170, 957 (1983).

${ }^{77}$ W. H. Taylor and P. J. Hagerman, J. Mol. Biol. 212, 363 (1990).

${ }^{78}$ The polymer chain also wanders transversely a bit but the associated entropy is entirely negligible in the asymptotic limit $\alpha \gg 1$.

${ }^{79}$ A. D. Bates and A. Maxwell, DNA Topology (IRL, Oxford, 1993).

${ }^{80}$ L. Onsager, Ann. N.Y. Acad. Sci. 51, 627 (1949).

${ }^{81}$ R. Eppenga and D. Frenkel, Mol. Phys. 34, 667 (1984).

${ }^{82}$ J. Torbet and E. Di Capua, EMBO J. 8, 4351 (1989).

${ }^{83}$ T. Odijk, Biophys. Chem. 46, 69 (1993).

${ }^{84}$ J. F. Marko and E. D. Siggia, Phys. Rev. E 52, 2912 (1995). In an interesting harmonic analysis, Marko and Siggia also conclude that the energy of tight bending is essentially independent of undulations. Since a naive expansion is not uniformly valid [see, e.g., Eqs. (3.11), (3.12), and (3.15)], I have attempted a Langer WKB approximation. This justifies a harmonic analysis and yields a second order entropy [Eqs. (2.15) and (3.37)] not contained in the harmonic approximation and signifying substantial thermal softening of the bend.

${ }^{85}$ A. Stroobants, H. N. W. Lekkerkerker, and T. Odijk, Macromolecules 19, 2232 (1986)

${ }^{86}$ C. J. Benham, Ann. Rev. Biophys. Biophys. Chem. 14, 23 (1985).

${ }^{87}$ F. B. Fuller, Proc. Natl. Acad. Sci. USA 68, 815 (1971).

${ }^{88}$ L. C. A. Groot, M. E. Kuil, J. C. Leyte, J. R. C. van der Maarel, R. K. Heenan, S. M. King, and G. Jannink, Liq. Cryst. 17, 263 (1994).

${ }^{89}$ V. I. Salyanov, A. T. Dembo, and Yu. M. Yevdokimov, Liq. Cryst. 9, 229 (1991).

${ }^{90}$ A. V. Vologodskii, S. D. Levene, K. V. Klenin, M. D. Frank-Kamenetskii, and N. R. Cozzarelli, J. Mol. Biol. 227, 1224 (1992)

${ }^{91}$ A. R. Khokhlov and A. N. Semenov, Physica A 108, 546 (1981).

${ }^{92}$ J. Straley, Phys. Rev. A 14, 1835 (1976).

${ }^{93}$ T. Odijk, J. Phys. Chem. 91, 6060 (1987). 
${ }^{94}$ D. H. van Winkle, M. W. Davidson, W. X. Chen, and R. L. Rill, Macromolecules 23, 4140 (1990).

${ }^{95}$ D. H. van Winkle, M. W. Davidson, and R. L. Rill, J. Chem. Phys. 97, 5641 (1992).
${ }^{96}$ R. D. Kamien and D. R. Nelson, Phys. Rev. Lett. 74, 2499 (1995).

${ }^{97}$ J. H. White, Am. J. Math. 91, 693 (1969).

${ }^{98}$ G. J. Vroege and T. Odijk, J. Chem. Phys. 87, 4223 (1987).

${ }^{99}$ M. Dijkstra and D. Frenkel, Phys. Rev. E 51, 5891 (1995). 